\title{
PROYECCION DE LA POBLACION NACIONAL EN 1975 Y 1980
}

312

por

\section{Antonio Rodríguez Socorro}

Jefe de la Sección de Estadística del Instituto, de Estudios de Administración Local

SUMARIO: I. LA PROYECCION DE LA POBLACION NACIONAL EN 1975 Y 1980: 1. Previsiones anteriores y la evolución Real. 2. PersPECTIVAS 3. INDICES DE FERTILIDAD. 4. INDICES DE MASCULINIDAd. 5. NaCimientos probables y grupos de edad. 6. Perspectivas de la poblaCIÓN DE 1975 A 1980.-II. HIPOTESIS Y METODOS: 1. INDICE DE FEcundidad. 2. Nacimientos probables. 3. Población de SETENTa y cinco A CIEN AÑOS POR GRUPOS DE EDAD. 4. HIPÓTESIS MÁS RAZONABLE.-III. LA POBLACION ACTIVA: 1. Hipótesis y mérodos. 2. TeNDENCIA dB LA POBLACIÓN ACTIVA EN ESPAÑA.-IV. TABLAS ESPECIFICAS: A 1. Conjunto de la población. Hipótesis sin emigración. A 2. Conjunto de la población. Hipótesis CON emigración. a 3. Perspectivas sin y CON EMIGRACIÓN POR GRUPOS. A 4. VARIANTES DE LA HIPÓTESIS MÁS RAzonable. A 5. Población activa. Perspectivas por SEXo y pOR EDad a PARTIR DEL 1-I-1965.-V. BIBLIOGRAFIA.

\section{LA PROYECCION DE LA POBLACION NACIONAL EN 1975 Y 1980}

Este trabajo sigue la metodología del desarrollado por el autor en (11, Bibliog.). Tengo la ilusión de que pueda ser útil al IEAL en sus secciones técnicas para la programación y colaboración en los Planes de Desarrollo y en la asistencia a los programas de actuación provinciales. 


\section{Previsiones anteriores y la evolución real}

Para la O. E. C. E. realizó el I. N. E. un estudio sobre la población futura, en primero de año, de los quinquenios comprendidos entre 1956 y 1976, ambos inclusive, y los resultados fueron publicados en (7, Bibliog.). Los errores observados en aquella proyección fueron mínimos.

Refiriéndome a los resultados obtenidos en este trabajo, se observa una fuerte desviación entre los fallecidos habidos y los calculados - tabla 1-, pero no deben sorprender porque, por un lado, las tablas de mortalidad para la población española de 1950 señalan una esperanza de vida de 62,10 años, y las tablas abreviadas, por el método de Wiesler, para 1960, la esperanza de vida es de 69,65 años, y, por otro lado, la intensa emigración del quinquenio 1960-65 elimina del conjunto de fallecidos los correspondientes a la masa emigrante.

Los nacimientos habidos y calculados difieren menos que los fallecidos y siguen una tendencia en progresión geométrica creciente de razón aproximada 1,01, e igual ocurre con la población total -tabla 2-.

T A B L A 1

FALLECIMIENTOS (1)

\begin{tabular}{|c|c|c|c|c|}
\hline Años & Calculados & Habidos (2) & Diferencia & $\begin{array}{c}\% \text { de } \\
\text { desviación }\end{array}$ \\
\hline 1959 & 353.893 & 264.497 & 89.396 & 25,26 \\
\hline 1960 & 359.197 & 262.260 & 96.937 & 26,29 \\
\hline 1961 & 364.619 & 256.394 & 108.225 & 29,68 \\
\hline 1962 & 370.110 & 271.591 & 98.519 & 26,62 \\
\hline 1963 & 375.695 & 275.448 & 100.247 & 26,68 \\
\hline 1965 & 386.966 & 267.407 & 119.559 & 30,90 \\
\hline 1970 & 448.881 & 280.170 & 168.711 & 37,58 \\
\hline 1973 & 471.325 & 296.524 & 174.801 & 37,09 \\
\hline 1974 & 478.395 & 294.567 (Prov.) & 183.828 & 38,43 \\
\hline
\end{tabular}

(1) Sin los nacidos muertos, muertos al nacer o antes de las primeras veinticuatro horas de vida.

(2) Fuente: Anuario Estadistico de España, 1974, I. N.E., Madrid, 1975. 
T A B L A 2

NACIMIENTOS (3)

\begin{tabular}{|c|c|c|c|c|}
\hline$A \bar{n} o s$ & Calculados & Habidos & Diferencia & $\begin{array}{c}\% \text { de } \\
\text { desviación }\end{array}$ \\
\hline 1959 & 648.976 & 647.160 & 1.816 & 0,28 \\
\hline 1960 & 655.616 & 654.537 & 1.079 & 0,16 \\
\hline 1961 & 662.297 & 645.613 & 16.684 & 2,52 \\
\hline 1962 & 669.043 & 649.680 & 19.363 & 2,89 \\
\hline 1963 & 675.874 & 662.437 & 13.437 & 1,99 \\
\hline 1965 & 689.391 & 667.749 & 21.642 & 3,13 \\
\hline 1970 & 723.861 & 656.102 & 67.759 & 9,36 \\
\hline 1973 & 745.577 & 666.336 & 79.241 & 10,63 \\
\hline 1974 & 753.033 & 678.049 & 74.984 & 9,96 \\
\hline
\end{tabular}

\section{PeRspectivas}

La base de partida para los cálculos de la población futura ha sido la población que aparece en el Censo de 1960, tomada del Avance de las clasificaciones de la población, publicada por el I. N. E., comprendiendo al territorio peninsular, Provincias insulares, Ceuta y Melilla.

Para el cálculo de la tabla A-1 se ha seguido el procedimiento expuesto por el señor Prieto Vilches (3, Bibliog., tabla núm. 4) y que constituirán las tablas 3 y 4 de éste, pero que únicamente senalo numéricamente para dejarlo en sus estrictos límites el comentario y uso del mismo. Así, nombraremos como tabla 3 la de los coeficientes de supervivencia correspondientes a varones, y como tabla 4 la de los coeficientes de supervivencia correspondientes a mujeres. Tengo a disposición de los intèresados copias de dichas tablas y añadiría explicación y uso de las mismas.

\section{INDICES DE FERTILIDAD}

Se tuvo en cuenta la fertilidad obtenida por el Instituto Nacional de Estadística para los distintos grupos de edades correspondientes a 1960, según consta en la

T A B L A 5

$\begin{array}{crrrcccc}\text { Grupos de edades } & 15-19 & 20-24 & 25-29 & 30-34 & 35-39 & 40-44 & 45-49 \\ \text { año } 1960 & 0,00944 & 0,10673 & 0,18806 & 0,142425 & 0,08004 & 0,02925 & 0.00313\end{array}$

(3) Nacidos vivos. 


\section{INDICES DE MASCULINIDAD}

Se ha calculado el índice de masculinidad con el promedio de cinco años, según datos del I. N. E., tal como se expone en la

T A B L A 6

$\begin{array}{ccc}\text { Años } & & \text { Masculinidad } \\ 1959 & & 0,512916 \\ 1960 & & 0,514371 \\ 1961 & & 0,513865 \\ 1962 & & 0,513753 \\ 1963 & & 0,513552\end{array}$

5. NACIMIENTOS PROBABLES Y GRUPOS DE EDAD

De cero a cuatro años, de cinco a setenta y cuatro años y de setenta y cinco y más años.

Según el esquema de LExis, si llamamos Lx a los vivientes en 31 de diciembre de una generación de nacidos durante el año $x$

$$
L_{x}=\frac{l_{x}+l_{x+1}}{2}
$$

siendo $\mathrm{l}_{\mathrm{x}}$ los individuos que han alcanzado la edad $\mathrm{x}$. De las tablas mortalidad de 1950 (utilizadas, no obstante, la desviación indicada en el punto 1.1.), se han tomado los valores de $l_{x}$ que corresponden en la tabla núm. 3. En la tercera parte, 3.1., se hace la hipótesis de mejora en la mortalidad. Con los datos hallados se proyectó la población de 1966 (1.1.), dividiendo la misma en tres grupos: De cero a cinco años, de cinco a setenta y cuatro años y de setenta y cinco al final.

Los vivientes en 31 de diciembre del primer grupo son:

$$
\begin{gathered}
\frac{\mathrm{L}_{0}}{100.000} \cdot \mathrm{N}_{1965}+\frac{\mathrm{L}_{1}}{100.000} \cdot \mathrm{N}_{1964}+\frac{\mathrm{L}_{2}}{100.000} \cdot \mathrm{N}_{1963}+ \\
\quad+\frac{\mathrm{L}_{3}}{100.000} \cdot \mathrm{N}_{1962}+\frac{\mathrm{L}_{4}}{100.000} \cdot \mathrm{N}_{1961}
\end{gathered}
$$

representando $\mathrm{Nx}$ los nacimientos en el año $\mathrm{x}$; obteniéndose la 
T A B L A 7 (4)

\begin{tabular}{|c|c|c|c|c|c|c|}
\hline \multicolumn{7}{|c|}{ VARONES } \\
\hline \multirow{2}{*}{$A \tilde{n} o s$} & \multirow{2}{*}{ Nacimientos } & $\mathrm{L}_{0} \cdot \mathrm{Nx}$ & $\mathrm{L}_{1} \cdot \mathrm{Nx}$ & $\mathrm{L}_{2} \cdot \mathrm{Nx}$ & $\mathrm{L}_{3} \cdot \mathrm{Nx}$ & $\mathrm{L}_{4} \cdot \mathrm{Nx}$ \\
\hline & & $10^{+5}$ & $10^{+5}$ & $10^{+5}$ & $10^{+5}$ & $1 U^{+3}$ \\
\hline $\begin{array}{llll}1966 & \ldots & \ldots & \ldots \\
1967 & \ldots & \ldots & \ldots \\
1968 & \ldots & \ldots & \ldots \\
1969 & \ldots & \ldots & . . \\
1970 & \ldots & \ldots & \ldots \\
1971 & \ldots & \ldots & \ldots\end{array}$ & $\begin{array}{l}334.440 \\
335.817 \\
337.194 \\
338.572 \\
339.950 \\
338.728\end{array}$ & 328.278 & 313.306 & 308.745 & 305.434 & 302.996 \\
\hline $\begin{array}{llll}1976 & \ldots & \ldots & \ldots \\
1981 & \ldots & \ldots & \ldots\end{array}$ & $\begin{array}{l}348.172 \\
361.374\end{array}$ & & & & & \\
\hline & & & ERES & & & \\
\hline $\begin{array}{llll}1966 & \ldots & \ldots & \ldots \\
1967 & \ldots & \ldots & \ldots \\
1968 & \ldots & \ldots & . . \\
1969 & \ldots & \ldots & \ldots \\
1970 & \ldots & \ldots & . . \\
1971 & \ldots & \ldots & . .\end{array}$ & $\begin{array}{l}316.613 \\
317.917 \\
319.221 \\
320.524 \\
321.829 \\
320.673\end{array}$ & 312.325 & 299.693 & 295.393 & 292.299 & 290.048 \\
\hline $\begin{array}{llll}1976 & \ldots & \ldots & \ldots \\
1981 & \ldots & \ldots & \ldots\end{array}$ & $\begin{array}{l}329.613 \\
342.112\end{array}$ & & & & & \\
\hline
\end{tabular}

La población de cinco a setenta y cuatro años en 1-I-1961 proviene de las generaciones que, en 1 de enero de 1961, estaban comprendidas entre cero y sesenta y nueve años.

Representando por $a_{n}$ a la suma de los vivientes (L) pertenecientes a este grupo, y $a_{n+1}$ a los del grupo siguiente (grupos de cin-

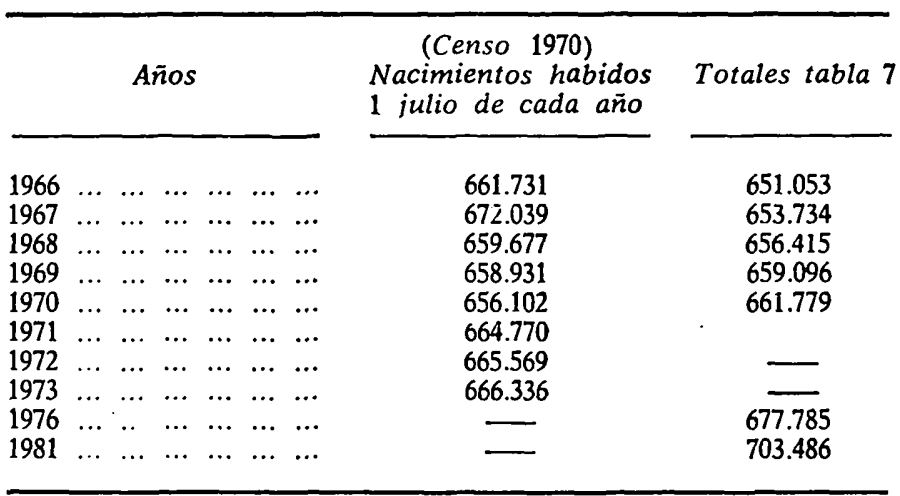

(4) La columna censal está tomada del Anuario Estadistico de España, 1974, capítulo II, tabla 2.1 , pág. 61 . 
co edades consecutivas), el cociente $a_{n+1} / a_{n}$, entre dos grupos consecutivos, es la probabilidad de que un individuo del primer grupo pertenezca al segundo, después de que han transcurrido cinco años de vida (tabla núm. 3). Multiplicando los distintos grupos de edades de 1 de enero de 1961 por su probabilidad respectiva, se obtiene la población de 1 de enero de 1966, comprendidos entre cinco y setenta y cuatro años (tabla núm. A-1).

El grupo de setenta y cinco y más años en 1 de enero de 1966 proviene de los que en 1960 tenían setenta-setenta y cuatro años, y de los ya existentes de setenta y cinco y más años.

Las Tablas de mortalidad de 1950 dan vivientes masculinos en 31 de diciembre hasta ciento un años, y femeninos hasta ciento dos. Por ello, se ha repartido el conjunto de setenta y cinco y más años de 1960 proporcionalmente a la población de 1950 (tabla núm. 8), y los vivientes de las edades noventa y cinco y noventa y nueve y cien más, teniendo en cuenta que, cinco años después, los vivientes mayores de noventa y siete años no existirán, con la hipótesis de las Tablas de mortalidad de 1950. De forma análoga se ha calculado la población femenina en 1 de enero de 1966, sólo que en el conjunto de setenta y cinco y más años se calcularon los vivientes de noventa y siete años (tablas núms. 3, 4, A-1, 7 y 8).

Partiendo de los datos de 1 de enero de 1966 y por análogas operaciones que las realizadas para la obtención de las cifras de dicho año, se consiguieron las de 1 de enero de 1971, y aplicado el método reiteradamente, las de 1 de enero de 1976 y 1 de enero de 1981, motivo por el cual van subrayados los valores en las tablas números A-1 y A-2. Las cifras intermedias se obtuvieron por interpolación geométrica.

T A L A 8

POBLACION: VARONES

\begin{tabular}{|c|c|c|c|c|}
\hline Grupos de edad & Censo 1960 & 1-I-1971 & 1-I-1976 & 1-I-1981 \\
\hline $\begin{array}{llllll}75-79 & \ldots & \ldots & \ldots & \ldots & \ldots \\
80-84 & \ldots & \ldots & \ldots & \ldots & \ldots \\
85-89 & \ldots & \ldots & \ldots & \ldots & \ldots \\
90-94 & \ldots & \ldots & \ldots & \ldots & \ldots \\
95-99 & \ldots & \ldots & \ldots & \ldots & \ldots \\
100 & \text { y } & \text { más } & \ldots & \ldots & \ldots\end{array}$ & $\begin{array}{r}189.409 \\
88.556 \\
31.385 \\
6.206 \\
1.394 \\
27\end{array}$ & $\begin{array}{r}207.590 \\
105.936 \\
32.226 \\
5.276 \\
411 \\
6\end{array}$ & $\begin{array}{r}240.891 \\
105.856 \\
35.319 \\
5.759 \\
386 \\
7\end{array}$ & $\begin{array}{r}259.095 \\
122.930 \\
35.293 \\
6.311 \\
422 \\
7\end{array}$ \\
\hline Totales $\ldots \ldots$ & 316.977 & 351.445 & 388.218 & 424.058 \\
\hline
\end{tabular}


POBLACION: MUJERES

\begin{tabular}{|c|c|c|c|c|}
\hline Grupos de edad & Censo 1960 & 1-I-1971 & 1-I-1976 & 1-I-1981 \\
\hline $\begin{array}{llllll}75-79 & \ldots & \ldots & \ldots & \ldots & \ldots \\
80-84 & \ldots & \ldots & \ldots & \ldots & \ldots \\
85-89 & \ldots & \ldots & \ldots & \ldots & \ldots \\
90-94 & \ldots & \ldots & \ldots & \ldots & \ldots \\
95-99 & \ldots & \ldots & \ldots & \ldots & \ldots \\
100 & \text { y } & \text { más } & \ldots & \ldots & \ldots \\
1\end{array}$ & $\begin{array}{r}279.483 \\
148.397 \\
59.238 \\
16.841 \\
4.921 \\
349\end{array}$ & $\begin{array}{r}317.365 \\
175.220 \\
58.708 \\
11.094 \\
984 \\
23\end{array}$ & $\begin{array}{r}358.648 \\
179.133 \\
65.210 \\
11.793 \\
917 \\
21\end{array}$ & $\begin{array}{r}377.068 \\
202.434 \\
66.666 \\
13.100 \\
975 \\
20\end{array}$ \\
\hline Totales $\ldots \ldots$ & 509.229 & 563.394 & 615.722 & 660.263 \\
\hline
\end{tabular}

\section{Perspectivas de la POBlación de 1975 a 1980}

Para la proyección de la población, teniendo en cuenta la emigración (tabla núm. A-2), se ha considerado el saldo emigratorio posible de 50.000 emigrantes por año, con las salvedades del caso, ya que estos fenómenos, sobre todo en un plazo más o menos largo, son difíciles de fijar apriorísticamente. Ese grupo de personas se ha repartido por grupos de edades, según los datos de emigrantes asistidos por el Instituto Español de Emigración en la emigración continental en los cinco últimos trimestres de 1964, constituyendo una muestra algo representativa de la emigración por grupo de edades, cuyos valores medios han sido expresados en la

T A B L A 9

GRUPOS DE EDAD, EN \%

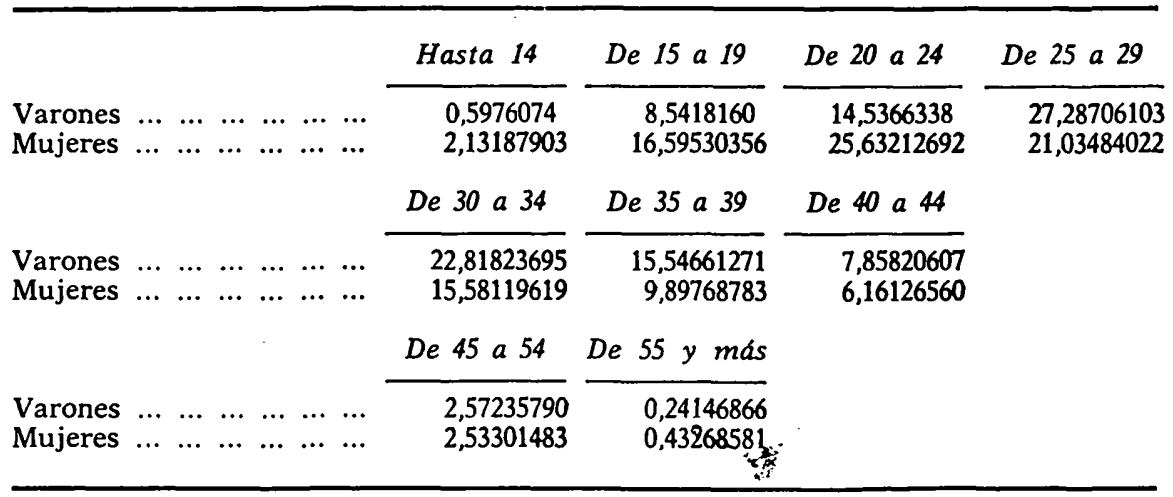


Hay que hacer una advertencia: En el reparto que se ha realizado para obtener la tabla A-2, teniendo en cuenta la tabla A-1, se comete un error, porque éste no refleja los saldos emigratorios, sino el número de emigrantes, y los que retornan vuelven más viejos; pero por carecer de otros datos fidedignos nos hemos visto obligados a utilizar éstos como único medio de comparación.

Suponiendo en los próximos años un saldo equivalente a los 50.000 individuos, corresponde disminuir en los años 1965 (1.1.), 1966 (1.1), 1967 (1.1), 1971 (1.1) y 1981 (1.1) el número de 200.000, $250.000,300.000,500.000$ y 1.000 .000 de personas, respectivamente.

\section{HIPOTESIS Y METODOS}

\section{INDICE DE FECUNDIDAD}

Para realizar la tabla A-3 consideramos como índice de fecundidad el promedio de los tres censos citados en la

T A B L A 10

\begin{tabular}{|c|c|c|c|c|c|c|c|}
\hline & $15-19$ & $20-24$ & $25-29$ & $30-34$ & $35-39$ & $40-44$ & $45-49$ \\
\hline $1940 \ldots \ldots \ldots \ldots$ & 0,00860 & 0,08964 & 0,18520 & 0,16620 & 0,10954 & 0,04393 & 0,00732 \\
\hline $1950 \ldots \ldots \ldots \ldots$ & 0,00788 & 0,08223 & 0,15390 & 0,12405 & 0,08453 & 0,03309 & 0,00444 \\
\hline $1960 \ldots \ldots \ldots \ldots$ & 0,00944 & 0,10673 & 0,18806 & 0,14245 & 0,08004 & 0,02925 & 0,00313 \\
\hline Promedio ... ... ... & 0,00864 & 0,09287 & 0,17572 & 0,14423 & 0,09137 & 0,03442 & 0,00496 \\
\hline
\end{tabular}

Se hace notar que los valores más probables para los grupos de edades de quince-diecinueve, veinte-veinticuatro, veinticincoveintinueve y treinta-treinta y cuatro seguirán una tendencia creciente respecto de 1960, ya que los valores de 1940 y 1950 vienen afectados por las anomalías derivadas de la guerra de Liberación y posguerra.

Como coeficiente de masculinidad se tomó el mismo que se utilizó para realizar el cuadro A-1.

Respecto de la mortalidad, calculamos el promedio de los tantos por ciento de desviación hallados, tal como sigue:

$$
1 / 5(25,26+26,99+29,68+26,62+26,68)=27,04
$$


y es por ello por lo que convenimos en mejorar la mortalidad en este valor.

Para ello construimos una tabla de coeficientes de supervivencia de forma análoga a las tablas núms. 3 y 4 , teniendo en cuenta la mejora de supervivencia, que se podría expresar así:

$$
(1-\mathrm{K}) 0,2704+\mathrm{K}=0,2704+0,7296 \mathrm{~K}
$$

dando lugar a los datos de la

T A B L A 11 (5)

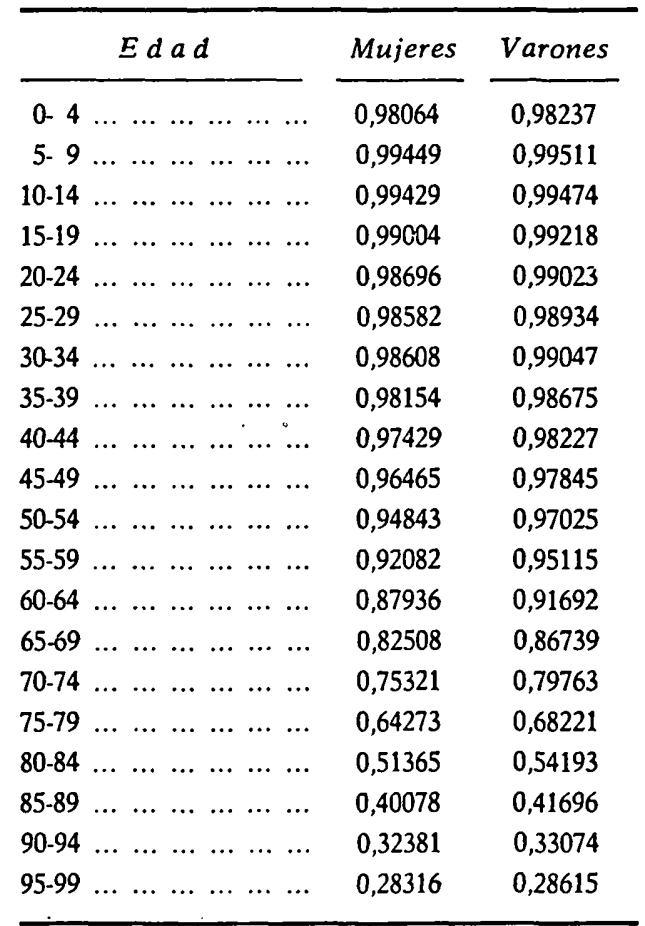

Para hallar los nacimientos probables y grupos de edad de cero a cuatro años, de cinco a setenta y cuatro años y de setenta y cinco y más años se empleó el mismo procedimiento que el utilizado para desarrollar la tabla A-1, con las nuevas hipótesis de fecundidad y mortalidad, obteniéndose los resultados siguientes (tablas números 12,13 y 14 ):

(5) En el Anuario Estadistico de España, 1974, I. N. E., 1975, en el cap. II, 1.2.2, señala a los supervivientes del Censo 1970 con cifras provisionales. 


\section{NACIMIENTOS PROBABLES}

T A B A 12

COEFICIENTE: 0,513691

\begin{tabular}{|c|c|c|c|}
\hline$A \bar{n} \circ \mathrm{s}$ & Nacimientos & Varones & Mujeres \\
\hline 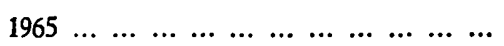 & 646.350 & 332.024 & 314.326 \\
\hline $\begin{array}{lllllllllll}1966 & \ldots & \ldots & \ldots & \ldots & \ldots & \ldots & \ldots & \ldots & \ldots & \ldots\end{array} \ldots$ & 648.344 & 333.048 & 315.296 \\
\hline $1967 \ldots \ldots \ldots$ & 650.338 & 334.073 & 316.265 \\
\hline $\begin{array}{llllllllllll} & 1968 & \ldots & \ldots & \ldots & \ldots & \ldots & \ldots & \ldots & \ldots & \ldots & \ldots\end{array}$ & 652.332 & 335.097 & 317.235 \\
\hline 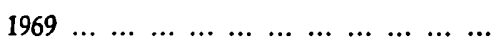 & 654.326 & 336.121 & 318.205 \\
\hline 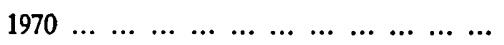 & 656.319 & 337.145 & 319.174 \\
\hline $\begin{array}{llllllllllll}1971 & \ldots & \ldots & \ldots & \ldots & \ldots & \ldots & \ldots & \ldots & \ldots & \ldots & \ldots\end{array}$ & 659.492 & 338.775 & 320.717 \\
\hline $\begin{array}{ccccccccc}1976 & \ldots & \ldots & \ldots & \ldots & \ldots & \ldots & \ldots & \ldots\end{array} \ldots$ & 677.573 & 348.063 & 329.510 \\
\hline $\begin{array}{llllllllllll}1981 & \ldots & \ldots & \ldots & \ldots & \ldots & \ldots & \ldots & \ldots & \ldots & \ldots & \ldots\end{array}$ & 699.133 & 359.138 & 339.995 \\
\hline
\end{tabular}

T A L A 13

VARONES

\begin{tabular}{|c|c|c|c|c|c|c|}
\hline & & $\mathrm{L}_{0} \cdot \mathrm{Nx}$ & $\mathrm{L}_{1} \cdot \mathrm{Nx}$ & $\mathrm{L}_{2} \cdot \mathrm{Nx}$ & $\mathrm{L}_{3} \cdot \mathrm{Nx}$ & $\mathrm{L}_{4} \cdot \mathrm{Nx}$ \\
\hline Años & Nacimientos & $10^{5}$ & $10^{5}$ & $10^{5}$ & $10^{3}$ & $10^{5}$ \\
\hline $\begin{array}{llll}1966 & \ldots & \ldots & \ldots \\
1967 & \ldots & \ldots & \ldots \\
1968 & \ldots & \ldots & \ldots \\
1969 & \ldots & \ldots & \ldots \\
1970 & \ldots & \ldots & \ldots \\
1971 & \ldots & \ldots & \ldots \\
1976 & \ldots & \ldots & \ldots \\
1981 & \ldots & \ldots & \ldots\end{array}$ & $\begin{array}{l}333.048 \\
334.073 \\
335.097 \\
336.121 \\
337.145 \\
338.775 \\
348.063 \\
359.158\end{array}$ & 328.700 & 317.819 & 314.468 & 312.021 & 310.201 \\
\hline
\end{tabular}

MUJERES

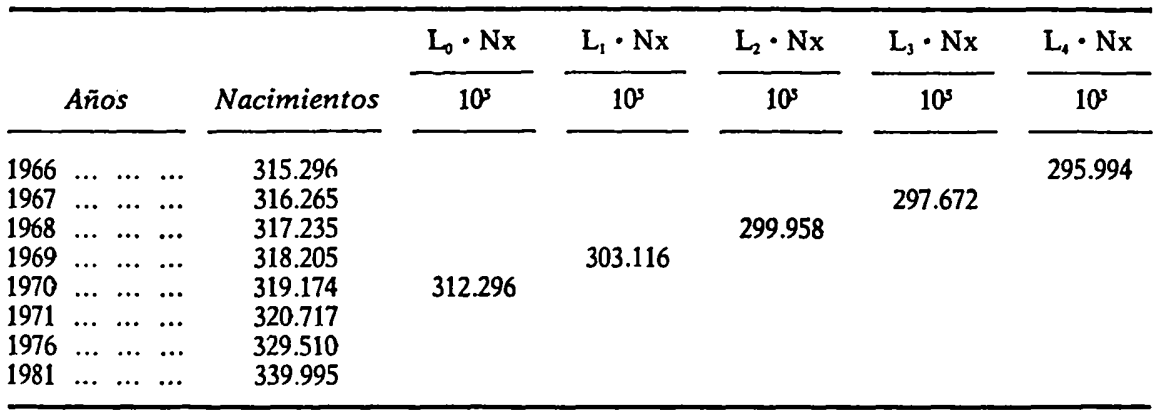


3. Población de SETENTA y CINCO a CIEN AÑOS POR GRUPOS DE EDAD

T A B L A 14.1

A) VARONES

\begin{tabular}{|c|c|c|c|c|}
\hline Grupos de edad & Censo 1960 & $1-I-1971$ & $1-I-1976$ & $1-I-1981$ \\
\hline $\begin{array}{llllll}75-79 & \ldots & \ldots & \ldots & \ldots & \ldots \\
80-84 & \ldots & \ldots & \ldots & \ldots & \ldots \\
85-89 & \ldots & \ldots & \ldots & \ldots & \ldots \\
90-94 & \ldots & \ldots & \ldots & \ldots & \ldots \\
95-99 & \ldots & \ldots & \ldots & \ldots & \ldots \\
100 & \ldots & \ldots & \ldots & \ldots & \ldots \\
10 & \ldots & \ldots\end{array}$ & $\begin{array}{r}189.409 \\
88.556 \\
31.385 \\
6.206 \\
1.394 \\
27\end{array}$ & $\begin{array}{r}256.233 \\
151.866 \\
62.531 \\
18.231 \\
4.073 \\
569\end{array}$ & $\begin{array}{r}313.506 \\
164.689 \\
78.006 \\
25.061 \\
5.903 \\
1.153\end{array}$ & $\begin{array}{r}348.299 \\
201.500 \\
84.593 \\
31.263 \\
8.115 \\
1.671\end{array}$ \\
\hline Total ...... & 316.977 & 493.503 & 588.318 & 675.441 \\
\hline
\end{tabular}

B) MUJERES

\begin{tabular}{|c|c|c|c|c|}
\hline Grupos de edad & Censo 1960 & $1-I-1971$ & 1.I-1976 & $I-I-198 I$ \\
\hline 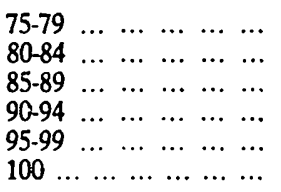 & $\begin{array}{r}279.483 \\
148.397 \\
59.238 \\
16.841 \\
4.921 \\
349\end{array}$ & $\begin{array}{r}371.344 \\
233.761 \\
103.328 \\
33.532 \\
8.169 \\
1.594\end{array}$ & $\begin{array}{r}434.231 \\
253.335 \\
126.682 \\
43.084 \\
11.090 \\
2.338\end{array}$ & $\begin{array}{r}465.388 \\
296.237 \\
137.290 \\
52.821 \\
14.250 \\
3.173\end{array}$ \\
\hline Total ...... & 509.229 & 751.728 & 870.760 & 969.159 \\
\hline
\end{tabular}

\section{HiPOTESIS MÁS RAZONABLE}

Según la referencia bibliográfica 10 , la población total en 1965 , 1970, 1975 y 1980 (a 1 de enero) con las dos hipótesis: 1) sin emigración, y 2) con emigración, y tasa anual de 50.000 personas, son las correspondientes a la

T A B L A 14.2

\begin{tabular}{|c|c|c|c|c|c|c|c|c|}
\hline \multirow{2}{*}{ Hipótesis } & \multicolumn{2}{|c|}{1965} & \multicolumn{2}{|c|}{1970} & \multicolumn{2}{|c|}{1975} & \multicolumn{2}{|c|}{1980} \\
\hline & Millones & Indice & Millones & Indice & Millones & Indice & Millones & Indice \\
\hline $\begin{array}{lllll}1 & \ldots & \ldots & \ldots & \ldots \\
2 & \ldots & \ldots & \ldots & \ldots\end{array}$ & $\begin{array}{l}31,58 \\
31,58\end{array}$ & $\begin{array}{l}100,0 \\
100,0\end{array}$ & $\begin{array}{l}32,84 \\
32,39\end{array}$ & $\begin{array}{l}104,0 \\
103,2\end{array}$ & $\begin{array}{l}34,06 \\
33,36\end{array}$ & $\begin{array}{l}107,7 \\
106,3\end{array}$ & $\begin{array}{l}35,25 \\
34,30\end{array}$ & $\begin{array}{l}111,6 \\
109,3\end{array}$ \\
\hline
\end{tabular}




\section{LA POBLACION ACTIVA}

\section{HIPótesis Y MÉTOdOS}

Las perspectivas de la población activa se indican en el cuadro C, y para su confección se tuvieron en cuenta las perspectivas con migraciones de la tabla A-2, los datos del Censo y los de la $T a-$ bla XIII del avance del Censo de 1960, distribuyéndose los individuos en la

$$
\text { T A B L A } 15.1
$$

\begin{tabular}{|c|c|c|c|c|c|c|c|c|}
\hline \multicolumn{3}{|c|}{ 1-I-1961 } & \multicolumn{3}{|c|}{ I-I-1965 } & \multicolumn{3}{|c|}{$1-1 \cdot 1970$} \\
\hline Pob. 15-64 & $\%$ & Pob.act. & Pob. 15-64 & $\%$ & Pob.act. & Pob. 15-64 & $\%$ & Pob. act. \\
\hline $\begin{array}{lr}\text { V. } & 9.485 \\
\text { M. } & 10.158\end{array}$ & $\begin{array}{r}100,29 \\
20,87\end{array}$ & $\begin{array}{l}9.514 \\
2.120\end{array}$ & $\begin{array}{lr}\text { V. } & 9.67 \mathrm{i} \\
\text { M. } & 10.429\end{array}$ & $\begin{array}{l}99,05 \\
22,92\end{array}$ & $\begin{array}{l}9.579 \\
2.390\end{array}$ & $\begin{array}{lr}\text { V. } & 9.921 \\
\text { M. } 10.717\end{array}$ & $\begin{array}{l}97,50 \\
25,49\end{array}$ & $\begin{array}{l}9.673 \\
2.732\end{array}$ \\
\hline \multicolumn{3}{|c|}{ I-I-1971 } & \multicolumn{3}{|c|}{ I-I-1976 } & \multicolumn{3}{|c|}{ 1-I-1981 } \\
\hline Pob. $15-64$ & $\%$ & Pob.act. & Pob. 15-64 & $\%$ & Pob.act. & Pob. 15-64 & $\%$ & Pob.act. \\
\hline $\begin{array}{lr}\text { V. } & 9.971 \\
\text { M. } & 10.777\end{array}$ & $\begin{array}{l}97,18 \\
26,00\end{array}$ & $\begin{array}{l}9.690 \\
2.802\end{array}$ & $\begin{array}{ll}\text { V. } 10.265 \\
\text { M. } 11.127\end{array}$ & $\begin{array}{l}95,63 \\
28,57\end{array}$ & $\begin{array}{l}9.816 \\
3.179\end{array}$ & $\begin{array}{ll}\text { V. } & 10.547 \\
\text { M. } & 11.447\end{array}$ & $\begin{array}{l}94,07 \\
31,13\end{array}$ & $\begin{array}{l}9.922 \\
3.564\end{array}$ \\
\hline
\end{tabular}

Si consideramos la referencia 10 (bibliografía), la población de edad activa, por sexos y por grandes grupos de edad, perspectivas sin emigración, obtendríamos para los años 1965, 1970, 1975 y 1980 (1 de enero) los datos de la

T A B L A 15.2

\begin{tabular}{|c|c|c|c|c|c|}
\hline \multirow{2}{*}{ Edad } & \multirow{2}{*}{ Sexo } & \multicolumn{2}{|c|}{1965} & \multicolumn{2}{|c|}{1970} \\
\hline & & Millones & Indice & Millones & Indice \\
\hline Menos de 45 años ...... & $\begin{array}{lll}\text { Varón } & \ldots & \ldots \\
\text { Mujer } & \ldots & \ldots \\
\text { V. }+ \text { M. } & \ldots & \ldots\end{array}$ & $\begin{array}{r}6,90 \\
7: 09 \\
13,99\end{array}$ & $\begin{array}{l}100,0 \\
100,0 \\
100,0\end{array}$ & $\begin{array}{r}7,16 \\
7,18 \\
14,34\end{array}$ & $\begin{array}{l}103,7 \\
101,2 \\
102,5\end{array}$ \\
\hline 45 años y más $\ldots \ldots \ldots$ & $\begin{array}{lll}\text { Varón } & \ldots & \ldots \\
\text { Mujer } & \ldots & \ldots \\
\text { V. }+ \text { M. } & \ldots & \ldots\end{array}$ & $\begin{array}{l}2,93 \\
2,67 \\
5,60\end{array}$ & $\begin{array}{l}100,0 \\
100,0 \\
100,0\end{array}$ & $\begin{array}{l}3,12 \\
2,85 \\
5,97\end{array}$ & $\begin{array}{l}106,5 \\
106,7 \\
106,6\end{array}$ \\
\hline Conjunto $\ldots \ldots \ldots \ldots$ & $\begin{array}{lll}\text { Varón } & \ldots & \ldots \\
\text { Mujer } & \ldots & \ldots \\
\text { V. }+ \text { M. } & \ldots & \ldots\end{array}$ & $\begin{array}{r}9,83 \\
9,76 \\
19,59\end{array}$ & $\begin{array}{l}100,0 \\
100,0 \\
100,0\end{array}$ & $\begin{array}{l}10,28 \\
10,03 \\
20,31\end{array}$ & $\begin{array}{l}104,6 \\
102,8 \\
103,7\end{array}$ \\
\hline Indice de población to & $\begin{array}{llll}\ldots & \ldots & \ldots & \ldots\end{array}$ & 一 & 100,0 & - & 104,0 \\
\hline
\end{tabular}


T A B L A 15.2 (Continuación)

\begin{tabular}{|c|c|c|c|c|c|}
\hline \multirow{2}{*}{ Edad } & \multirow{2}{*}{ Sexo } & \multicolumn{2}{|c|}{1975} & \multicolumn{2}{|c|}{1980} \\
\hline & & Millones & Indice & Millones & Indice \\
\hline Menos de 45 años ...... & $\begin{array}{lll}\text { Varón } & \ldots & \ldots \\
\text { Mujer } & \ldots & \ldots \\
\text { V. + M. } & \ldots & \ldots\end{array}$ & $\begin{array}{r}7,39 \\
7,35 \\
14,74\end{array}$ & $\begin{array}{l}107,1 \\
103,7 \\
105,4\end{array}$ & $\begin{array}{r}7,64 \\
7,51 \\
15,15\end{array}$ & $\begin{array}{l}110,7 \\
105,9 \\
108,3\end{array}$ \\
\hline 45 años y más ... ... ... & $\begin{array}{lll}\text { Varón } & \ldots & \ldots \\
\text { Mujer } & \ldots & \ldots \\
\text { V. }+ \text { M. } & \ldots & \ldots\end{array}$ & $\begin{array}{l}3,37 \\
3,04 \\
6,41\end{array}$ & $\begin{array}{l}115,0 \\
113,9 \\
114,5\end{array}$ & $\begin{array}{l}3,61 \\
3,24 \\
6,85\end{array}$ & $\begin{array}{l}123,2 \\
121,3 \\
122,3\end{array}$ \\
\hline Conjunto $\ldots \ldots \ldots c c c c c$ & $\begin{array}{lll}\text { Varón } & \ldots & \ldots \\
\text { Mujer } & \ldots & \ldots \\
\text { V. }+ \text { M. } & \ldots & \ldots\end{array}$ & $\begin{array}{r}10,76 \\
: \quad 10,39 \\
\quad 21,15\end{array}$ & $\begin{array}{l}109,5 \\
106,5 \\
108,0\end{array}$ & $\begin{array}{l}11,25 \\
10,75 \\
22,60\end{array}$ & $\begin{array}{l}114,5 \\
110,1 \\
112,3\end{array}$ \\
\hline Indice de población total & $\begin{array}{llllll} & \ldots & \ldots & \ldots & \ldots & \ldots\end{array}$ & - & 107,7 & - & 111,6 \\
\hline
\end{tabular}

De 1965 a 1971 las perspectivas de la población activa se establecieron teniendo en cuenta la corrección global de 50.000 individuos como saldo emigratorio negativo.

En un trabajo a desarrollar en el futuro se harán las correcciones en la población activa a partir de 1970, teniendo en cuenta:

a) Aumento en la duración de la escolaridad y distintas procedencias escolares (6).

b) Variación eventual en la edad de retiro.

c) Variación del empleo de las mujeres, y

d) Otras causas a precisar en la evolución de la actividad.

Teniendo en cuenta la clasificación de la población activa por ramas de actividad prevista para 1967 y 1970, teniendo en cuenta el Plan de Desarrollo Económico se obtuvo la siguiente tabla:

(6) Entradas en la población activa para 197475 en miles: primaria $(288,1)$; media elemental $(31,0)$; media superior: técnica $(48,3)$ y general $(80,2)$; superior (25), según Las necesidades de educación y el desarrollo económico-social de España, Ministerio de Educación Nacional, O. C. D. E., Madrid, diciembre 1963. 
T A B L A 16 (7)

POBLACION (EN MILES)

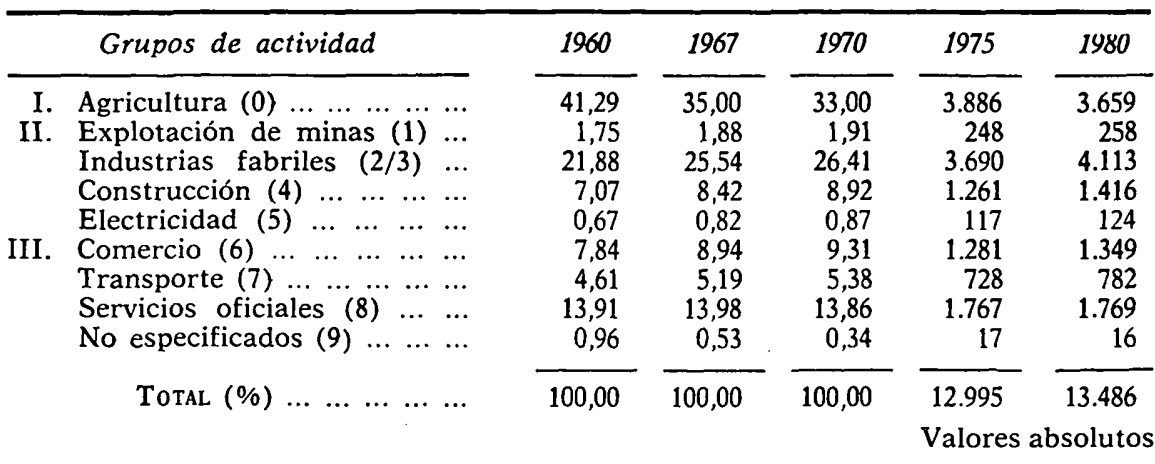

\section{Tendencia de la población activa en España}

Se observó que las variaciones de un Censo, con relación al siguiente, tenían una tendencia definida.

Estas variaciones se estudiaron no solamente para el total, sino también para cada una de las agrupciones de la clasificación nacional de Actividades Económicas.

Los datos básicos se obtuvieron de los Censos de Población de los años referidos:

T A B L A 16 bis

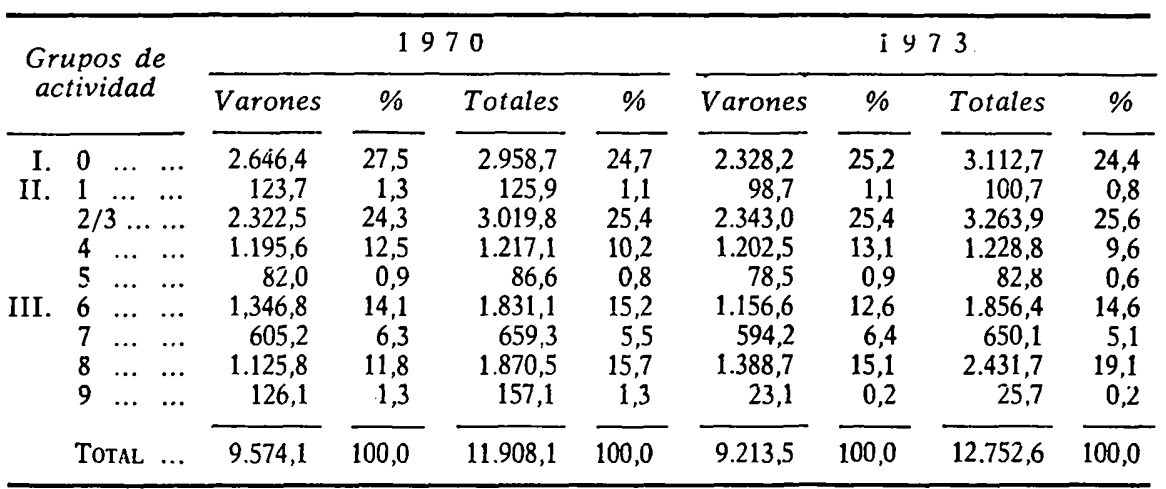

(7) Se podría ampliar la tabla 16 con las clasificaciones censales del Anuario Estadístico de España, 1974, capítulo II, tabla 1.1.3.1, pág. 52, en valores absolutos para varones y total, en 1970, y con la referencia 14, pág. 165, para el segundo semestre de 1973, obteniendo la tabla 16 bis. 
T A B L A 17

\begin{tabular}{|c|c|c|c|c|c|c|}
\hline $\begin{array}{l}\text { Grupos de } \\
\text { actividades }\end{array}$ & 1920 & 1930 & 1940 & 1950 & 1960 & 1970 \\
\hline $\begin{array}{lllllll}0 & \ldots & \ldots & \ldots & \ldots & \ldots\end{array}$ & $4.555,6$ & $4.691,7$ & $4.781,0$ & $5.271,0$ & $4.803,3$ & $2.958,7$ \\
\hline $\begin{array}{llllll}1 & \ldots & \ldots & \ldots & \ldots & \ldots\end{array}$ & 132,2 & 114,8 & 99,6 & 173,8 & 203,5 & 125,9 \\
\hline $\begin{array}{lllll}2 / 3 & \ldots & \ldots & \ldots & \ldots\end{array}$ & $1.324,1$ & $1.599,5$ & $1.695,0$ & $1.904,0$ & $2.545,9$ & $3.019,8$ \\
\hline $\begin{array}{llllll}4 & \ldots & \ldots & \ldots & \ldots & \ldots\end{array}$ & 216,3 & 284,9 & 373,4 & 574,3 & 822,1 & $1.217,1$ \\
\hline $\begin{array}{llllll}5 & \ldots & \ldots & \ldots & \ldots & \ldots\end{array}$ & 21,7 & 23,7 & 44,0 & 56,5 & 80,8 & 86,6 \\
\hline $\begin{array}{llllll}6 & \ldots & \ldots & \ldots & \ldots & \ldots\end{array}$ & 385,1 & 469,5 & 589,0 & 697,7 & 911,8 & $1.813,1$ \\
\hline $\begin{array}{llllll}7 & \ldots & \ldots & \ldots & \ldots & \ldots\end{array}$ & 222,8 & 293,0 & 310,2 & 421,3 & 536,5 & 659,3 \\
\hline $\begin{array}{llllll}8 & \ldots & \ldots & \ldots & \ldots & \ldots\end{array}$ & 842,3 & $1.095,9$ & $1.316,6$ & $1.522,5$ & $1.618,6$ & $1.870,5$ \\
\hline $\begin{array}{llllll}9 & \ldots & \ldots & \ldots & \ldots & \ldots\end{array}$ & 262,3 & 199,5 & 10,9 & 172,0 & 111,7 & 157,1 \\
\hline Total $\ldots \ldots$ & $7.962,4$ & $8.772,5$ & $9.219,7$ & $10.793,1$ & $11.634,2$ & $11.908,1$ \\
\hline
\end{tabular}

FUENTE: Anuarios del I. N. E.

con cuyos datos se obtuvieron los siguientes porcentajes:

T A B L A 18

PORCENTAJES DE CADA CENSO CON RESPECTO AL CENSO ANTERIOR

\begin{tabular}{|c|c|c|c|c|c|}
\hline Grupos de actividades & $1930 / 1920$ & $1940 / 1930$ & $1950 / 1940$ & $1960 / 1950$ & $1970 / 1960$ \\
\hline $\begin{array}{llllllllll}0 & \ldots & \ldots & \ldots & \ldots & \ldots & \ldots & \ldots & \ldots\end{array}$ & 102,99 & 101,90 & 110,25 & 91,12 & 61,60 \\
\hline $\begin{array}{llllllllll}1 & \ldots & \ldots & \ldots & \ldots & \ldots & \ldots & \ldots & \ldots & \ldots\end{array}$ & 86,84 & 86,76 & 174,50 & 117,69 & 61,87 \\
\hline $\begin{array}{lllllllll}2 / 3 & \ldots & \ldots & \ldots & \ldots & \ldots & \ldots & \ldots & \ldots\end{array}$ & 120,80 & 105,97 & 112,33 & 133,71 & 118,61 \\
\hline $\begin{array}{llllllllll}4 & \ldots & \ldots & \ldots & \ldots & \ldots & \ldots & \ldots & \ldots & \ldots\end{array}$ & 131,72 & 131,06 & 153,80 & 143,15 & 148,05 \\
\hline $\begin{array}{llllllllll}5 & \ldots & \ldots & \ldots & \ldots & \ldots & . . & \ldots & \ldots & \ldots\end{array}$ & 109,22 & 185,65 & 128,41 & 143,01 & 107,18 \\
\hline $\begin{array}{llllllllll}6 & \ldots & \ldots & \ldots & \ldots & \ldots & \ldots & \ldots & \ldots & \ldots\end{array}$ & 121,92 & 125,45 & 118,46 & 130,69 & 198,85 \\
\hline $\begin{array}{llllllllll}7 & \ldots & \ldots & \ldots & \ldots & \ldots & \ldots & \ldots & \ldots & \ldots\end{array}$ & 131,51 & 105,87 & 135,82 & 127,34 & 122,89 \\
\hline $\begin{array}{llllllllll}8 & \ldots & \ldots & \ldots & \ldots & \ldots & \ldots & \ldots & \ldots & \ldots\end{array}$ & 130,11 & 120,14 & 115,64 & 106,31 & 115,56 \\
\hline $\begin{array}{llllllllll}9 & \ldots & \ldots & \ldots & \ldots & \ldots & \ldots & \ldots & \ldots & \ldots\end{array}$ & 76.06 & 5,56 & $1.577,98$ & 64,94 & 140,64 \\
\hline Total $(\%) \ldots \ldots \ldots$ & 110,17 & 105,10 & 117,07 & 107,79 & 102,35 \\
\hline
\end{tabular}

Resultando las previsiones, con base, 1960: 
T A B L A 19 (8)

\begin{tabular}{|c|c|c|c|c|}
\hline Agrupaciones & 1960 & 1962 & 1967 & 1970 \\
\hline 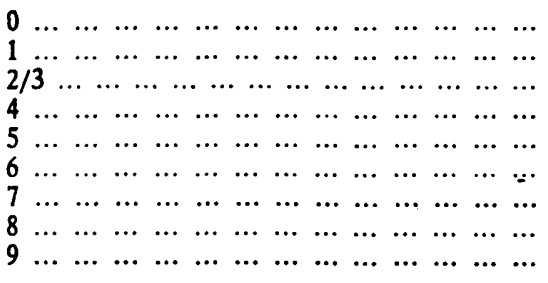 & $\begin{array}{r}4.803,3 \\
203,5 \\
2.545,9 \\
822,1 \\
80,8 \\
911,8 \\
536,5 \\
1.618,6 \\
112,0\end{array}$ & $\begin{array}{r}4.300,6 \\
302,5 \\
3.582,6 \\
1.197,1 \\
99,9 \\
1.194,4 \\
725,0 \\
1.706,2 \\
118,0\end{array}$ & $\begin{array}{r}3.812,6 \\
323,6 \\
4.089,4 \\
1.180,5 \\
77,6 \\
1.241,1 \\
773,8 \\
1,649,2 \\
116,0\end{array}$ & $\begin{array}{r}3.467,7 \\
337,1 \\
4.448,9 \\
1.165,7 \\
61,0 \\
1.275,3 \\
809,1 \\
1.616,1 \\
120,0\end{array}$ \\
\hline $\begin{array}{ccccccccccccc}\mathrm{T}_{1} & \ldots & \ldots & \ldots & \ldots & \ldots & \ldots & \ldots & \ldots & \ldots & \ldots & \ldots & \ldots\end{array}$ & $11.634,5$ & $13.226,3$ & $13.263,8$ & $13.300,9$ \\
\hline \multirow[t]{2}{*}{ 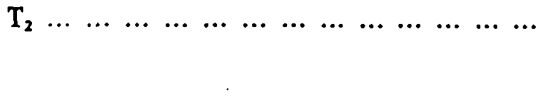 } & $11.634,5$ & $12.329,9$ & $12.457,7$ & $12.746,4$ \\
\hline & 1960 & 1962 & 1967 & 1970 \\
\hline 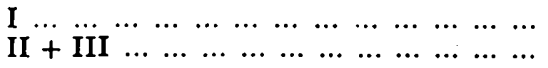 & $\begin{array}{l}4.803,3 \\
6.831,2\end{array}$ & $\begin{array}{l}4.300,6 \\
8.925,7\end{array}$ & $\begin{array}{l}3.812,6 \\
9.451,2\end{array}$ & $\begin{array}{l}3.467,7 \\
9.833,2\end{array}$ \\
\hline $\begin{array}{lllllllll}\text { Total } & \ldots & \ldots & \ldots & \ldots & \ldots & \ldots & \ldots & \ldots\end{array}$ & $11.634,5$ & $13.226,4$ & $13.263,8$ & $13.300,9$ \\
\hline
\end{tabular}

entre las cuales figura la agrupación 9 con datos que, aumentando sucesivamente, lo hacen dentro de límites prudentes.

El total por suma se ha consignado $T_{1}, y$ el total calculado mediante el ajuste parabólico figura con $\mathrm{T}_{2}$; la relación
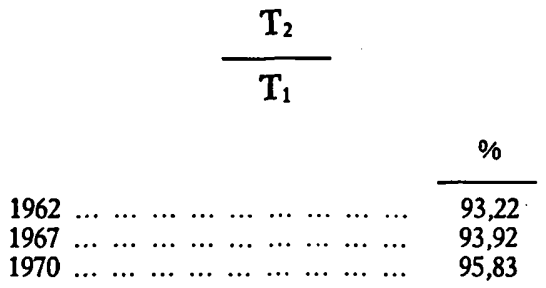

Calculando los porcentajes con cada año de cada uno de los totales obtenidos por suma se obtienen los siguientes resultados:

(8) Teniendo en cuenta los Censos de 1920, 1930, 1940, 1950 y 1960, resultaron, para cada grupo de actividad las parábolas siguientes:

Grupos

$0 \ldots y=-4,51 x^{2}+19,82 x+85,83$
$1 \ldots \ldots y=0,87 x^{2}+13,75 x+75,61$
$2, \ldots \ldots y=9,05 x^{2}-40,75 x+152,19$
$4 \ldots . y=-2,50 x^{2}+18,23 x+2.113 .10$
$5 \ldots . . . y=-15,46 x^{2}+81,70 x+53,25$

\section{Grupos}

$6 \ldots . . y=2,18 x^{2}-8,94 x+130,18$
$7 \ldots . . y=4,29 x^{2}-19,71 x+142,23$
$8 \ldots . y=0,16 x^{2}-8,39 x+137,83$
TотаL $y=-1,05 x^{2}+5,75 x+103,56$


T A B L A 20

\begin{tabular}{|c|c|c|c|c|}
\hline Agrupaciones & 1960 & 1962 & 1967 & 1970 \\
\hline 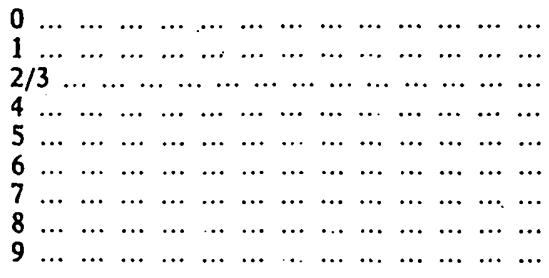 & $\begin{array}{r}41,28 \\
1,75 \\
21,88 \\
7,07 \\
0,70 \\
7,84 \\
4,61 \\
13,91 \\
0,96\end{array}$ & $\begin{array}{r}32,52 \\
2,28 \\
27,09 \\
9,05 \\
0,76 \\
9,03 \\
5,48 \\
12,90 \\
0,89\end{array}$ & $\begin{array}{r}28,71 \\
2,44 \\
30,80 \\
8,89 \\
0,58 \\
9,35 \\
5,83 \\
12,42 \\
0,98\end{array}$ & $\begin{array}{r}26,04 \\
2,53 \\
33,41 \\
8,75 \\
0,46 \\
9,58 \\
6,68 \\
12,14 \\
1,01\end{array}$ \\
\hline & 100,00 & 100,00 & 100,00 & 100,00 \\
\hline
\end{tabular}

que indican la variación prevista de la estructura de la población activa en los años reseñados y con la hipótesis de que dicha variación se realice de acuerdo con tendencias de los porcentajes de cada censo, con relación al anterior, según los cálculos realizados, y cuyos resultados se expresan a continuación:

T A B L A 21

\begin{tabular}{|c|c|c|c|c|}
\hline Agrupaciones & $\begin{array}{c}\% \text { de } 1960 \\
\text { con relacion } \\
\text { a } 1950\end{array}$ & $\begin{array}{c}\% \text { de } 1962 \\
\text { con relación } \\
\text { a } 1960\end{array}$ & $\begin{array}{c}\% \text { de } 1967 \\
\text { con relación } \\
\text { a } 1960\end{array}$ & $\begin{array}{c}\% \text { de } 1970 \\
\text { con relación } \\
\text { a } 1960\end{array}$ \\
\hline 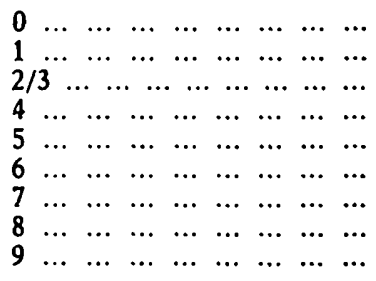 & $\begin{array}{r}91,12 \\
117,89 \\
133,71 \\
143,15 \\
143,01 \\
130,69 \\
127,34 \\
106,31 \\
64,94\end{array}$ & $\begin{array}{r}89,54 \\
148,67 \\
140,72 \\
145,61 \\
123,72 \\
130,99 \\
135,14 \\
105,41 \\
105,36\end{array}$ & $\begin{array}{r}79,38 \\
159,41 \\
160,62 \\
143,61 \\
95,79 \\
136,19 \\
144,37 \\
101,93 \\
116,00\end{array}$ & $\begin{array}{r}72,20 \\
166,06 \\
174,74 \\
141,81 \\
75,32 \\
139,84 \\
150,95 \\
99,88 \\
114,32\end{array}$ \\
\hline $\begin{array}{llllllllll}\mathrm{T}_{1} & \ldots & \ldots & \ldots & \ldots & \ldots & \ldots & \ldots & \ldots\end{array}$ & 109,48 & 113,68 & 114,00 & 120,00 \\
\hline $\begin{array}{llllllllll}\mathrm{T}_{2} & \ldots & \ldots & \ldots & \ldots & \ldots & \ldots & \ldots & \ldots\end{array}$ & 107,19 & 105,98 & 107,08 & 109,56 \\
\hline
\end{tabular}

Utilizando ecuaciones que se han deducido a base de esos datos se han calculado los valores teóricos correspondientes a los años: $1920,1930,1940,1950,1960,1962,1967$ у 1970 con los siguientes resultados: 
T A B L A 22

\begin{tabular}{|c|c|c|c|c|c|c|c|}
\hline $\begin{array}{l}\text { Grupos de } \\
\text { actividades }\end{array}$ & 1930 & 1940 & 1950 & 1960 & 1962 & 1967 & 1970 \\
\hline 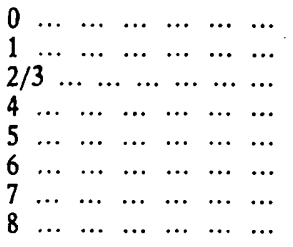 & $\begin{array}{r}101,14 \\
90,23 \\
120,49 \\
128,83 \\
119,50 \\
123,41 \\
126,80 \\
129,60\end{array}$ & $\begin{array}{l}107,44 \\
106,58 \\
106,89 \\
139,57 \\
154,82 \\
120,99 \\
119,98 \\
121,69\end{array}$ & $\begin{array}{l}104,71 \\
124,67 \\
111,40 \\
145,31 \\
159,23 \\
122,93 \\
121,72 \\
114,10\end{array}$ & $\begin{array}{r}92,97 \\
144,50 \\
134,02 \\
146,06 \\
132,73 \\
129,21 \\
132,05 \\
106,83\end{array}$ & $\begin{array}{r}89,53 \\
148,67 \\
140,72 \\
145,62 \\
123,72 \\
130,99 \\
135,14 \\
105,41\end{array}$ & $\begin{array}{r}79,37 \\
159,40 \\
160,62 \\
143,61 \\
94,78 \\
136,19 \\
144,38 \\
161,93\end{array}$ & $\begin{array}{r}72,20 \\
160,06 \\
174,74 \\
141,82 \\
75,32 \\
139,84 \\
150,95 \\
99,88\end{array}$ \\
\hline $\begin{array}{llllllll}\mathrm{T} & \ldots & \ldots & \ldots & \ldots & \ldots & \ldots\end{array}$ & 108,25 & 110,839 & 111,324 & 109,705 & 109,128 & 107,32 & 105,982 \\
\hline
\end{tabular}

Existen dos resultados para el total de población activa: uno corresponde al resultado de la extrapolación de la ecuación, que se refiere a la población total activa, y otro a la suma de las poblaciones calculadas para cada uno de los sectores.

Aplicando los porcentajes de distribución de cada año y las poblaciones activas totales calculadas, resultarian las siguientes cantidades:

T A B L A 23

\begin{tabular}{|c|c|c|c|}
\hline Grupos de actividades & 1962 & 1967 & 1970 \\
\hline 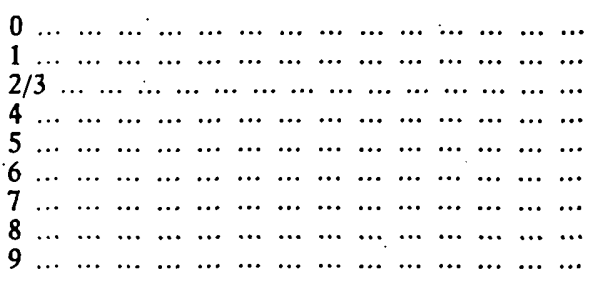 & $\begin{array}{r}4.009,7 \\
281,1 \\
3.340,2 \\
1.115,9 \\
95,7 \\
1.113,4 \\
675,7 \\
1.590,5 \\
109,7\end{array}$ & $\begin{array}{r}3.576,6 \\
304,0 \\
3.837,0 \\
1.107,5 \\
72,2 \\
1.164,8 \\
726,3 \\
1.547,2 \\
122,1\end{array}$ & $\begin{array}{r}3.319,2 \\
322,5 \\
4.258,6 \\
1.115,3 \\
58,6 \\
1.221,1 \\
775,6 \\
1.547,4 \\
128,7\end{array}$ \\
\hline 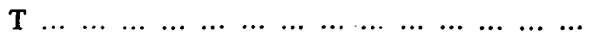 & $12.329,9$ & $12.457,7$ & $12.746,4$ \\
\hline 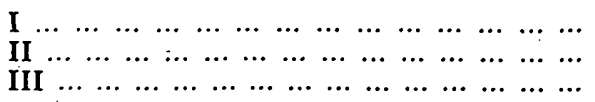 & $\begin{array}{l}4.009,7 \\
4.830,9 \\
3.489,3\end{array}$ & $\begin{array}{l}3.576,6 \\
5.320,7 \\
3.560,4\end{array}$ & $\begin{array}{l}3.319,2 \\
5.755,0 \\
3.672,2\end{array}$ \\
\hline 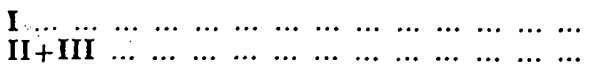 & $\begin{array}{l}4.009,7 \\
8.320,2\end{array}$ & $\begin{array}{l}3.576,6 \\
8.881,1\end{array}$ & $\begin{array}{l}3.319,2 \\
9.427,2\end{array}$ \\
\hline
\end{tabular}

Se consideran como válidas estas estimaciones, ya que la ecuación correspondiente a la población activa total se entiende como una ecuación de condición. 
REVL-1976, núm. 189. RODRIGUEZ SOCORRO, ANTONIO. PROYECCION DE LA POBLACION NACI...

IV. TABLAS ESPECIFICAS

A 1. Conjunto de la población: Hipótesis SIN emigración (PERSPECTIVA POR SEXo y por edad a partiR Del 1 DE ENERo De 1965. PeRsPeCtiva SIN EMigRACIÓN)

D A T O D D ORIGE N (1-I-1961)

\begin{tabular}{|c|c|c|c|c|c|c|c|c|c|c|}
\hline \multirow{2}{*}{ Edad } & \multicolumn{2}{|c|}{$1-1 \cdot 1961$} & \multicolumn{2}{|c|}{$1-1-1965$} & \multicolumn{2}{|c|}{$1-1-1970$} & \multicolumn{2}{|c|}{$31-X I I-1975$} & \multicolumn{2}{|c|}{$31-X I I-1980$} \\
\hline & Varones & Mujeres & Varones & Mujeres & Varones & Mujeres & Varones & Mujeres & Varones & Mujeres \\
\hline $0-4 \ldots \ldots \ldots \ldots$ & 1.541 .887 & 1.481 .114 & 1.548 .829 & 1.481 .786 & 1.557 .121 & 1.488 .198 & 1.587 .569 & 1.518 .435 & 1.640 .269 & 1.567 .639 \\
\hline $\begin{array}{llllll}5 & \ldots & \ldots & \ldots & \ldots & \ldots\end{array}$ & 289.504 & 267.580 & 297.537 & 284.121 & 302.799 & 290.235 & 304.741 & 291.796 & 310.373 & 297.413 \\
\hline $\begin{array}{llllll}6 & \ldots & \ldots & \ldots & \ldots & \ldots\end{array}$ & 277.655 & 258.332 & 296.849 & 283.544 & 302.099 & 289.646 & 304.036 & 291.204 & 309.655 & 296.810 \\
\hline $\begin{array}{llllll}7 & \ldots & \ldots & \ldots & \ldots & \ldots\end{array}$ & 283.232 & 261.422 & 296.236 & 283.042 & 301.476 & 289.133 & 303.409 & 290.688 & 309.017 & 296.284 \\
\hline $\begin{array}{llllll}8 & \ldots & \ldots & \ldots & \ldots & \ldots\end{array}$ & 284.831 & 260.799 & 295.695 & 282.604 & 300.925 & 288.685 & 302.854 & 290.238 & 308.451 & 295.825 \\
\hline $\begin{array}{llllll}9 & \ldots & \ldots & \ldots & \ldots & \ldots\end{array}$ & 268.534 & 248.177 & 295.219 & 282.217 & 300.440 & 288.290 & 302.366 & 289.842 & 307.955 & 295.421 \\
\hline $\begin{array}{llllll}5-9 & \ldots & \ldots & \ldots & \ldots & \ldots\end{array}$ & 1.403 .756 & 1.296 .310 & 1.481 .536 & 1.415.528 & 1.507 .739 & 1.445 .989 & 1.517 .406 & 1.453 .768 & 1.545 .451 & 1.481 .753 \\
\hline $\begin{array}{lllllll}10 & \ldots & \ldots & \ldots & \ldots & \ldots\end{array}$ & 257.115 & 250.101 & 277.084 & 258.798 & 294.796 & 281.867 & 300.346 & 287.965 & 301.933 & 289.482 \\
\hline $\begin{array}{llllll}11 & \ldots & \ldots & \ldots & \ldots & \ldots\end{array}$ & 271.546 & 265.102 & 276.724 & 258.498 & 294.412 & 281.539 & 299.955 & 287.630 & 301.540 & 289.145 \\
\hline $\begin{array}{lllllll}12 & \ldots & \ldots & \ldots & \ldots & \ldots\end{array}$ & 289.684 & 282.176 & 276.391 & 258.202 & 294.058 & 281.218 & 299.594 & 287.302 & 301.178 & 288.814 \\
\hline $\begin{array}{lllllll}13 & \ldots & \ldots & \ldots & \ldots & \ldots\end{array}$ & 262.668 & 255.776 & 276.074 & 257.899 & 293.721 & 280.888 & 299.251 & 286.965 & 300.832 & 288.476 \\
\hline $\begin{array}{lllllll}14 & \ldots & \ldots & \ldots & \ldots & \ldots\end{array}$ & 256.409 & 251.197 & 275.734 & 257.574 & 293.358 & 280.534 & 298.882 & 286.603 & 300.462 & 288.112 \\
\hline $\begin{array}{lllll}10-14 & \ldots & \ldots & \ldots & \ldots\end{array}$ & 1.337 .422 & 1.304 .352 & 1.382 .007 & 1.290 .971 & 1.470 .345 & 1.406 .046 & 1.498 .028 & 1.436 .465 & 1.505 .945 & 1.444.029 \\
\hline $\begin{array}{lllllll}15 & \ldots & \ldots & \ldots & \ldots & \ldots\end{array}$ & 263.518 & 274.961 & 258.841 & 257.258 & 275.296 & 257.207 & 296.775 & 286.039 & 298.446 & 286.201 \\
\hline $\begin{array}{lllllll}16 & \ldots & \ldots & \ldots & \ldots & \ldots\end{array}$ & 251.477 & 262.431 & 258.397 & 256.859 & 274.825 & 256.808 & 296.266 & 285.595 & 297.934 & 285.757 \\
\hline $\begin{array}{llllll}17 & \ldots & \ldots & \ldots & \ldots & \ldots\end{array}$ & 251.469 & 261.303 & 257.883 & 256.420 & 274.278 & 256.368 & 295.678 & 285.106 & 297.342 & 285.268 \\
\hline $\begin{array}{lllllll}18 & \ldots & \ldots & \ldots & \ldots & \ldots\end{array}$ & 216.300 & 223.522 & 257.299 & 255.940 & 273.656 & 255.889 & 295.007 & 284.572 & 296.668 & 284.734 \\
\hline $\begin{array}{lllllll}19 & \ldots & \ldots & \ldots & \ldots & \ldots\end{array}$ & 199.743 & 207.460 & 256.645 & 255.422 & 272.962 & 255.371 & 294.259 & 283.996 & 295.914 & 284.157 \\
\hline $\begin{array}{lllll}15-19 & \ldots & \ldots & \ldots & \ldots\end{array}$ & 1.182 .507 & 1.229 .677 & 1.289 .065 & 1.281 .899 & 1.317.017 & 1.281 .643 & 1.477 .985 & 1.425 .308 & 1.486 .304 & 1.426 .117 \\
\hline
\end{tabular}




\begin{tabular}{|c|c|c|c|c|c|c|c|c|c|c|}
\hline \multirow{2}{*}{ Edad } & \multicolumn{2}{|c|}{$1-I-1961$} & \multicolumn{2}{|c|}{$1-I-1965$} & \multicolumn{2}{|c|}{$1-1-1970$} & \multicolumn{2}{|c|}{$31-X I I-1975$} & \multicolumn{2}{|c|}{$31-X I I-1980$} \\
\hline & Varones. & Mujeres & Varones & Mujeres & Varones & Mujeres & Varones & Mujeres & Varones & Mujeres \\
\hline $20 \ldots \ldots \ldots$ & 271.967 & 262.047 & 233.529 & 239.917 & 257.452 & 254.815 & 274.430 & 254.163 & 293.438 & 283.381 \\
\hline $\begin{array}{lllllll}21 & \ldots & \ldots & \ldots & \ldots & \ldots\end{array}$ & 168.151 & 162.634 & 232.824 & 239.364 & 256.675 & 254.227 & 273.602 & 253.576 & 292.552 & 282.727 \\
\hline $\begin{array}{llllll}22 & \ldots & \ldots & \ldots & \ldots & \ldots\end{array}$ & 211.986 & 204.539 & 232.073 & 238.781 & 255.846 & 253.608 & 272.719 & 252.958 & 291.608 & 282.038 \\
\hline $23 \ldots \ldots \ldots$ & 231.300 & 226.467 & 231.284 & 238.172 & 254.977 & 252.962 & 271.793 & 252.313 & 290.617 & 281.319 \\
\hline $24 \ldots \ldots \ldots$ & 252.034 & 247.179 & 230.466 & 237.542 & 254.075 & 252.293 & 270.832 & 251.646 & 289.590 & 280.514 \\
\hline $20-24 \ldots \ldots \ldots$ & 1.135 .438 & 1.102 .866 & 1.160 .176 & 1.193.776 & 1.279 .025 & 1.267 .905 & 1.363 .376 & 1.264 .656 & 1.457 .805 & 1.410 .039 \\
\hline $\begin{array}{lllll}25.29 & \ldots & \ldots & \ldots & \ldots\end{array}$ & 1.192 .438 & 1.252 .616 & 1.130 .608 & 1.121 .005 & 1.139.378 & 1.176 .903 & 1.285 .451 & 1.263 .930 & 1.339 .015 & 1.247 .723 \\
\hline $\begin{array}{lllll}30-34 & \ldots & \ldots & \ldots & \ldots\end{array}$ & 1.176 .649 & 1.183 .898 & 1.170 .734 & 1.224.232 & 1.108 .235 & 1.102 .813 & 1.123 .259 & 1.182 .681 & 1.260 .459 & 1.249 .464 \\
\hline $\begin{array}{lllll}35-39 & \ldots & \ldots & \ldots & \ldots\end{array}$ & 1.077 .941 & 1.158 .575 & 1.138 .943 & 1.166 .461 & 1.148 .387 & 1.208 .079 & 1.072 .600 & 1.058 .196 & 1.101 .813 & 1.167 .231 \\
\hline $40-44 \ldots \ldots c c$ & 849.343 & 964.636 & 1.010 .404 & 1.102 .936 & 1.109 .719 & 1.145 .247 & 1.117 .922 & 1.196 .043 & 1.045 .463 & 1.038 .957 \\
\hline $45-49 \ldots \ldots \ldots$ & 821.837 & 929.085 & 819.902 & 938.770 & 971.425 & 1.073 .870 & 1.085 .356 & 1.119.307 & 1.078 .535 & 1.166 .974 \\
\hline $\begin{array}{ccccc}50-54 & \ldots & \ldots & \ldots & \ldots\end{array}$ & 782.512 & 879.632 & 782.114 & 897.243 & 780.171 & 911.022 & 964.535 & 1.077 .085 & 1.032 .765 & 1.086 .250 \\
\hline $\begin{array}{llllll}55.59 & \ldots & \ldots & \ldots & \ldots\end{array}$ & 692.147 & 771.291 & 720.189 & 829.267 & 726.851 & 860.593 & 724.598 & 876.148 & 896.356 & 1.033.164 \\
\hline $60-64 \ldots \ldots \ldots$ & 573.678 & 684.499 & 608.359 & 712.624 & 641.880 & 773.281 & 647.865 & 806.977 & 645.958 & 817.492 \\
\hline $65-69 \ldots \ldots \ldots$ & 412.309 & 536.736 & 465.515 & 592.592 & 507.553 & 631.360 & 541.079 & 697.628 & 540.736 & 715.087 \\
\hline $70.74 \ldots \ldots \ldots$ & 313.700 & 429.588 & 313.510 & 437.262 & 353.298 & 484.319 & 391.533 & 521.801 & 411.360 & 570.829 \\
\hline 75 y más ........ & 316.977 & 509.229 & 335.279 & 531.272 & 349.003 & 558.071 & 388.218 & 615.722 & 424.038 & 660.263 \\
\hline TOTAL ... ... & 4.810.541 & 15.714 .104 & 15.357 .170 & 16.217 .624 & 16.021 .147 & 16.815.339 & 16.786 .780 & 17.514 .150 & 17.412 .292 & 18.079 .011 \\
\hline
\end{tabular}


A 2. CONJUNTO DE LA POBlaCiÓN: HIPÓTESIS CON EMIGRACIÓN (PERSPECTIVAS POR SEXO Y POR EDAD A PARTIR DE 1 DE ENERo de 1965: PeRsPeCtiva CON EMIGRACión) (tasa neta, 50.000)

D A T O D E ORIGE N (1-I-1961)

\begin{tabular}{|c|c|c|c|c|c|c|c|c|c|c|}
\hline \multirow{2}{*}{ Edad } & \multicolumn{2}{|c|}{$1-I-1961$ (en miles) } & \multicolumn{2}{|c|}{$1-I-1965$} & \multicolumn{2}{|c|}{ 1-I-1970 } & \multicolumn{2}{|c|}{$31 \cdot X I I \cdot 1975$} & \multicolumn{2}{|c|}{$31-X I I-1980$} \\
\hline & Varones & Mujeres & Varones & Mujeres & Varones & Mujeres & Varones & Mujeres & Varones & Mujeres \\
\hline $04 \ldots \ldots \ldots$ & 1.542 & 1.481 & 1.548 .492 & 1.481 .489 & 1.556 .378 & 1.487 .553 & 1.586 .327 & 1.517 .352 & 1.638 .591 & 1.566 .176 \\
\hline $\begin{array}{lllllll} & \ldots & \ldots & \ldots & \ldots & \ldots\end{array}$ & 290 & 268 & 297.472 & 284.064 & 302.655 & 290.109 & 304.503 & 291.588 & 310.055 & 297.136 \\
\hline $\begin{array}{llllll}6 & \ldots & \ldots & \ldots & \ldots & \ldots\end{array}$ & 278 & 258 & 296.784 & 283.487 & 301.956 & 289.520 & 303.798 & 290.996 & 309.338 & 296.533 \\
\hline $\begin{array}{llllll}7 & \ldots & \ldots & \ldots & \ldots & \ldots\end{array}$ & 283 & 261 & 296.172 & 282.985 & 301.333 & 289.008 & 303.172 & 290.481 & 308.702 & 296.008 \\
\hline $\begin{array}{llllll}8 & \ldots & \ldots & \ldots & \ldots & \ldots\end{array}$ & 285 & 261 & 295.631 & 282.547 & 300.782 & 288.560 & 302.617 & 290.031 & 308.135 & 295.549 \\
\hline $\begin{array}{llllll}9 & \ldots & \ldots & \ldots & \ldots & \ldots\end{array}$ & 268 & 248 & 295.515 & 282.161 & 300.298 & 288.165 & 302.129 & 289.635 & 307.640 & 295.145 \\
\hline $\begin{array}{ccccccc}5-9 & \ldots & \ldots & \ldots & \ldots & \ldots\end{array}$ & 1.404 & 1.296 & 1.481 .214 & 1.415 .244 & 1.507 .024 & 1.445 .362 & 1.516 .219 & 1.452 .731 & 1.543 .870 & 1.480 .371 \\
\hline $10 \ldots \ldots \ldots \ldots$ & 257 & 250 & 277.024 & 258.746 & 294.656 & 281.743 & 300.111 & 287.760 & 301.624 & 289.212 \\
\hline $11 \ldots \ldots \ldots \ldots$ & 271 & 265 & 276.664 & 258.446 & 294.272 & 281.416 & 299.721 & 287.425 & 301.231 & 288.875 \\
\hline $12 \ldots \ldots \ldots \ldots$ & 290 & 282 & 276.330 & 258.150 & 293.918 & 281.095 & 299.360 & 287.097 & 300.870 & 288.545 \\
\hline $\begin{array}{llllll}13 & \ldots & \ldots & \ldots & \ldots & \ldots\end{array}$ & 263 & 256 & 276.014 & 257.847 & 293.521 & 280.765 & 299:017 & 286.760 & 300.524 & 288.207 \\
\hline $14 \ldots \ldots \ldots c c$ & 256 & 251 & 275.674 & 257.523 & 293.218 & 280.411 & 298.648 & 286.399 & 300.155 & 287.843 \\
\hline $10-14 \ldots \ldots \ldots$ & 1.337 & 1.304 & 1.381 .706 & 1.290 .712 & 1.469 .645 & 1.405 .430 & 1.496 .857 & 1.435 .441 & 1.504 .404 & 1.442 .682 \\
\hline $\begin{array}{llllll}15 & \ldots & \ldots & \ldots & \ldots & \ldots\end{array}$ & 264 & 275 & 256.085 & 255.949 & 269.129 & 254.267 & 286.440 & 281.129 & 284.666 & 279.655 \\
\hline $16 \ldots \ldots \ldots \ldots$ & 252 & 262 & 255.646 & 255.552 & 268.669 & 253.872 & 285.949 & 280.693 & 284.177 & 279.221 \\
\hline $\begin{array}{lllllll}17 & \ldots & \ldots & \ldots & \ldots & \ldots\end{array}$ & 251 & 261 & 255.137 & 255.115 & 268.134 & 253.437 & 285.381 & 280.212 & 283.613 & 278.743 \\
\hline $\begin{array}{llllll}18 & \ldots & \ldots & \ldots & \ldots & \ldots\end{array}$ & 216 & $224^{\circ}$ & 254.559 & 254.637 & 267.526 & 252.964 & 284.733 & 279.687 & 282.970 & 278.221 \\
\hline $19 \ldots \ldots \ldots \ldots$ & 200 & 208 & 253.912 & 254.122 & 266.848 & 252.452 & 284.011 & 279.122 & 282.250 & 277.657 \\
\hline $\begin{array}{ccccc}15-19 & \ldots & \ldots & \ldots & \ldots\end{array}$ & 1.183 & 1.230 & 1.275.339 & 1.275 .375 & 1.340 .306 & 1.226 .992 & 1.426 .514 & 1.400 .843 & 1.417 .676 & 1.393 .497 \\
\hline
\end{tabular}




\begin{tabular}{|c|c|c|c|c|c|c|c|c|c|c|}
\hline \multirow{2}{*}{ Edad } & \multicolumn{2}{|c|}{ 1-I-1961 (en miles) } & \multicolumn{2}{|c|}{ 1-I-1965 } & \multicolumn{2}{|c|}{$1-I-1970$} & \multicolumn{2}{|c|}{$31-X I I-1975$} & \multicolumn{2}{|c|}{$31-X I I-1980$} \\
\hline & Varones & Mujeres & Varones & Mujeres & Varones & Mujeres & Varones & Mujeres & Varones & Mujeres \\
\hline $20 \ldots \ldots \ldots$ & 272 & 262 & 228.827 & 237.892 & 247.137 & 250.312 & 256.798 & 246.569 & 269.928 & 273.256 \\
\hline $\begin{array}{lllllll}21 & \ldots & \ldots & \ldots & \ldots & \ldots\end{array}$ & 168 & 163 & 228.136 & 237.344 & 246.391 & 249.734 & 256.023 & 246.000 & 269.113 & 272.625 \\
\hline $\begin{array}{lllllll}22 & \ldots & \ldots & \ldots & \ldots & \ldots \\
22 & \ldots & \ldots & \ldots & \ldots\end{array}$ & 212 & 205 & 227.401 & 236.765 & 245.596 & 249.126 & 255.197 & 245.400 & 268.245 & 271.961 \\
\hline $\begin{array}{llllll}23 & \ldots & \ldots & \ldots & \ldots & \ldots\end{array}$ & 231 & 226 & 226.627 & 236.162 & 244.762 & 248.491 & 254.330 & 244.775 & 267.334 & 271.268 \\
\hline $\begin{array}{llllll}24 & \ldots & \ldots & \ldots & \ldots & \ldots\end{array}$ & $25 \%$ & 247 & 225.826 & 235.537 & 243.896 & 247.834 & 253.431 & 244.127 & 266.389 & 270.549 \\
\hline $20-24 \ldots \ldots c c c c$ & 1.135 & 1.103 & 1.136 .817 & 1.183 .700 & 1.227 .782 & 1.245 .497 & 1.275 .779 & 1226.871 & 1.341 .009 & 1.359 .659 \\
\hline $\begin{array}{ccccc}25-29 & \ldots & \ldots & \ldots & \ldots\end{array}$ & 1.192 & 1.253 & 1.086 .761 & 1.112 .736 & 1.040 .789 & 1.159.188 & 1.121.024 & 1.232 .922 & 1.119.779 & 1.206 .379 \\
\hline $\begin{array}{ccccc}30-34 & \ldots & \ldots & \ldots & \ldots\end{array}$ & 1.177 & 1.184 & 1.134 .068 & 1.218.107 & 1.026 .126 & 1.090 .843 & 985.760 & 1.159.713 & 1.077.127 & 1.214 .840 \\
\hline $\begin{array}{lllll}35-39 & \ldots & \ldots & \ldots & \ldots\end{array}$ & 1.078 & 1.159 & 1.113 .961 & 1.162 .571 & 1.092 .182 & 1.199 .485 & 978.919 & 1.043 .607 & 976.905 & 1.147.779 \\
\hline $40-44 \ldots \ldots \ldots$ & 849 & 965 & 997.777 & 1.100 .513 & 1.081 .717 & 1.139 .803 & 1.070 .570 & 1.186 .959 & 982.327 & 1.026 .845 \\
\hline $\begin{array}{ccccc}45-49 & \ldots & \ldots & \ldots & \ldots\end{array}$ & 822 & 929 & 817.786 & 938.261 & 968.613 & 1.072 .677 & 1.077 .149 & 1.117.404 & 1.067 .977 & 1.164 .397 \\
\hline $\begin{array}{llllll}50-54 & \ldots & \ldots & \ldots & \ldots\end{array}$ & 783 & 880 & 780.096 & 896.757 & 777.913 & 910.010 & 957.241 & 1.075 .254 & 1.022 .655 & 1.083 .851 \\
\hline $\begin{array}{llllll}55-59 & \ldots & \ldots & \ldots & \ldots\end{array}$ & 692 & 771 & 720.074 & 829.221 & $\mathbf{7 2 4 . 7 4 7}$ & 859.637 & 724.206 & 875.988 & 895.779 & 1.032 .932 \\
\hline $60-64 \ldots \ldots \ldots$ & 574 & 684 & 608.262 & 712.585 & 640.022 & 772.422 & 647.514 & 806.829 & 645.542 & 817.309 \\
\hline $\begin{array}{ccccc}65-69 & \ldots & \ldots & \ldots & \ldots\end{array}$ & 412 & 537 & 465.440 & 592.559 & 506.084 & 630.658 & 540.786 & 697.500 & 540.388 & 714.926 \\
\hline $\begin{array}{lllll}70-74 & \ldots & \ldots & \ldots & \ldots\end{array}$ & 314 & 430 & 313.460 & 437.238 & 352.276 & 483.781 & 391.321 & 521.705 & 411.095 & 570.701 \\
\hline 75 y más ........ & 317 & 509 & 225.228 & 531.245 & 347.993 & 557.451 & 388.008 & 615.510 & 423.720 & 660.115 \\
\hline TOTAL ... ... & 14.811 & 15.715 & 15.196 .481 & 16.178 .313 & 15.659 .597 & 16.726 .889 & 16.184 .194 & 17.366 .629 & 16.608 .844 & 17.882 .459 \\
\hline
\end{tabular}




\section{A 3. PERSPECTIVAS SIN Y CON EMIGRACIÓN POR GRUPOS}

A 3.1. PERSPECTIVAS SIN EMIGRACION. DATOS BASICOS. 1.-I-1961

\begin{tabular}{|c|c|c|c|c|c|c|c|c|c|c|c|}
\hline \multirow[b]{2}{*}{$A \tilde{n} o s$} & \multirow[b]{2}{*}{ Sexo } & \multicolumn{2}{|c|}{$1-I-1961$} & \multicolumn{2}{|c|}{$1-1-1965$} & \multicolumn{2}{|c|}{ I-I-1970 } & \multicolumn{2}{|c|}{$31-X I I-1975$} & \multicolumn{2}{|c|}{$31-X 11-1980$} \\
\hline & & $\begin{array}{l}\text { Núms. } \\
\text { absolts. }\end{array}$ & $\begin{array}{c}R . p o r \\
1.000\end{array}$ & $\begin{array}{l}\text { Nuims. } \\
\text { absolts. }\end{array}$ & $\begin{array}{c}R . p o r \\
1.000\end{array}$ & $\begin{array}{l}\text { Núms. } \\
\text { absolts. }\end{array}$ & $\begin{array}{l}R . \text { por } \\
1.000\end{array}$ & $\begin{array}{l}\text { Núms. } \\
\text { absolts. }\end{array}$ & $\begin{array}{c}R . p o r \\
1.000\end{array}$ & $\begin{array}{l}\text { Nums. } \\
\text { absolts. }\end{array}$ & $\begin{array}{c}R . p o r \\
1.000\end{array}$ \\
\hline $\begin{array}{cccccc}\text { Menos } & \text { de } & 15 & \ldots & \ldots \\
15-44 & \ldots & \ldots & \ldots & \ldots & \ldots \\
15-44 & \ldots & \ldots & \ldots & \ldots & \ldots \\
45-64 & \ldots & \ldots & \ldots & \ldots & \ldots \\
45-59 & \ldots & \ldots & \ldots & \ldots & \ldots \\
65 & \text { y más } & \ldots & \ldots & \ldots & \ldots \\
60 & \text { y } & \text { más } & \ldots & \ldots & \ldots \\
\end{array}$ & $\begin{array}{l}M+F \\
M \\
F \\
M \\
F \\
M \\
F\end{array}$ & $\begin{array}{l}8.364 \\
6.614 \\
6.894 \\
2.871 \\
2.580 \\
1.043 \\
2.160\end{array}$ & $\begin{array}{r}274,00 \\
216,67 \\
225,83 \\
94,05 \\
84,52 \\
34,17 \\
70,76\end{array}$ & $\begin{array}{l}8.602 \\
6.900 \\
7.090 \\
2.930 \\
2.665 \\
1.114 \\
2.274\end{array}$ & $\begin{array}{r}272,43 \\
218,52 \\
224,55 \\
92.80 \\
84,80 \\
35,28 \\
72,02\end{array}$ & $\begin{array}{l}8.874 \\
7.155 \\
7.183 \\
3.121 \\
2.846 \\
1.210 \\
2.447\end{array}$ & $\begin{array}{r}270,26 \\
217,90 \\
218,76 \\
95,05 \\
86,67 \\
36,85 \\
74,51\end{array}$ & $\begin{array}{l}9.011 \\
7.440 \\
7.391 \\
3.423 \\
3.072 \\
1.321 \\
2.643\end{array}$ & $\begin{array}{r}262,71 \\
216,90 \\
215,48 \\
99,79 \\
89,56 \\
38,51 \\
77,05\end{array}$ & $\begin{array}{l}9.185 \\
7.691 \\
7.535 \\
3.654 \\
3.286 \\
1.376 \\
2.764\end{array}$ & $\begin{array}{r}258,80 \\
216,70 \\
212,31 \\
102,96 \\
92,58 \\
38,77 \\
77,88\end{array}$ \\
\hline TOTAL $\ldots \ldots$ & & 30.526 & $1.000,00$ & 31.575 & $1.000,00$ & 32.836 & $1.000,00$ & 34.301 & $1.000,00$ & 35.491 & $1.000,00$ \\
\hline
\end{tabular}

\section{A 3.2. PERSPECTIVAS CON EMIGRACION}

\begin{tabular}{|c|c|c|c|c|c|c|c|c|c|c|c|}
\hline \multirow[b]{2}{*}{ Años } & \multirow[b]{2}{*}{ Sexo } & \multicolumn{2}{|c|}{$1-I-1961$} & \multicolumn{2}{|c|}{ I-I-1965 } & \multicolumn{2}{|c|}{ I-I-1970 } & \multicolumn{2}{|c|}{$31-X I I-1975$} & \multicolumn{2}{|c|}{$31 \cdot X I I \cdot 1980$} \\
\hline & & $\begin{array}{l}\text { Núms. } \\
\text { absolts. }\end{array}$ & $\begin{array}{c}R . \text { por } \\
1.000\end{array}$ & $\begin{array}{l}\text { Nums. } \\
\text { absolts. }\end{array}$ & $\begin{array}{c}R . p o r \\
1.000\end{array}$ & $\begin{array}{l}\text { Núms. } \\
\text { absolts. }\end{array}$ & $\begin{array}{c}R . p o r \\
1.000\end{array}$ & $\begin{array}{l}\text { Nums. } \\
\text { absolts. }\end{array}$ & $\begin{array}{c}R . p o r \\
1.000\end{array}$ & $\begin{array}{l}\text { Núms. } \\
\text { absolts. }\end{array}$ & $\begin{array}{c}\text { R. por } \\
1.000\end{array}$ \\
\hline 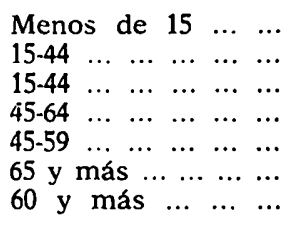 & $\begin{array}{l}M+F \\
M \\
F \\
M \\
F \\
M \\
F\end{array}$ & $\begin{array}{l}8.364 \\
6.614 \\
6.894 \\
2.871 \\
2.580 \\
1.043 \\
2.160\end{array}$ & $\begin{array}{r}274,00 \\
216,67 \\
225,83 \\
94,05 \\
84,52 \\
34,17 \\
70,76\end{array}$ & $\begin{array}{l}8.599 \\
6.745 \\
7.053 \\
2.926 \\
2.664 \\
1.114 \\
2.274\end{array}$ & $\begin{array}{r}274,06 \\
214,97 \\
224,80 \\
93,27 \\
84,92 \\
35,51 \\
72,47\end{array}$ & $\begin{array}{l}8.872 \\
6.809 \\
7.102 \\
3.111 \\
2.842 \\
1.206 \\
2.444\end{array}$ & $\begin{array}{r}273,92 \\
210,24 \\
219,28 \\
96,07 \\
87,76 \\
37,25 \\
75,48\end{array}$ & $\begin{array}{l}9.065 \\
6.859 \\
7.251 \\
3.406 \\
3.069 \\
1.320 \\
2.641\end{array}$ & $\begin{array}{r}268,40 \\
204,42 \\
216,12 \\
101,52 \\
91,46 \\
39,35 \\
78,43\end{array}$ & $\begin{array}{l}9.176 \\
6.915 \\
7.349 \\
3.632 \\
3.281 \\
1.375 \\
2.763\end{array}$ & $\begin{array}{r}266,04 \\
200,49 \\
213,07 \\
105,30 \\
95,13 \\
39,86 \\
80,11\end{array}$ \\
\hline TOTAL $\ldots \ldots$ & & 30.526 & $1.000,00$ & 31.375 & $1.000,00$ & 32.386 & $1.000,00$ & $33.551^{\prime}$ & $1.000,00$ & 34.491 & $1.000,00$ \\
\hline
\end{tabular}




\section{A 4. VARIANTES DE LA hipótesis MÁS RAZONABLE (clasificación por grupos de edades)}

\begin{tabular}{|c|c|c|c|c|c|c|c|c|c|c|c|c|}
\hline \multirow{3}{*}{ Años } & \multicolumn{4}{|c|}{ 1-1-1970. Modificacion } & \multicolumn{4}{|c|}{ 31-XII-1975. Modificación } & \multicolumn{4}{|c|}{ 31-XII-1980. Modificación } \\
\hline & \multicolumn{2}{|c|}{+} & \multicolumn{2}{|c|}{-} & \multicolumn{2}{|c|}{+} & \multicolumn{2}{|c|}{-} & \multicolumn{2}{|c|}{+} & \multicolumn{2}{|c|}{-} \\
\hline & Hombs. & Mujers. & Hombs. & Mujers. & Hombs. & Mujers. & Hombs. & Mujers. & Hombs. & Mujers. & Hombs. & Mujers. \\
\hline $0-4 \ldots \ldots \ldots$ & 24 & 18 & - & - & 28 & 22 & - & - & 31 & 25 & - & - \\
\hline $\begin{array}{llll}50-54 & \ldots & \ldots & \ldots\end{array}$ & 17 & 13 & - & - & 39 & 22 & - & - & 37 & 26 & - & - \\
\hline $\begin{array}{lllll}55-59 & \ldots & \ldots & \ldots\end{array}$ & 23 & 15 & - & - & 32 & 24 & - & - & 47 & 33 & - & - \\
\hline $60-64 \ldots \ldots \ldots$ & 32 & 23 & - & - & 44 & 32 & - & - & 51 & 38 & - & - \\
\hline $\begin{array}{lllll}65-69 & \ldots & \ldots & \ldots\end{array}$ & 41 & 32 & - & - & 60 & 46 & - & - & 68 & 54 & - & - \\
\hline $70-74 \ldots \ldots$ & 48 & 35 & - & - & 70 & 61 & - & - & 85 & 75 & - & - \\
\hline 75 y más ... & 130 & 172 & - & - & 200 & 255 & - & - & 251 & 309 & - & - \\
\hline
\end{tabular}

ObSERVACIONES: El sentido y la magnitud (en \%) de la diferencia entre la hipótesis más razonable y las variantes es: a) fecundidad, promedio censos 1940-1956-1960; $b$ ) mortalidad, disminución en 27,04 por 100 . 
REVL-1976, núm. 189. RODRIGUEZ SOCORRO, ANTONIO. PROYECCION DE LA POBLACION NACI...

A 5. Población ACtiva. PERSPECtivas POR SEXo Y POR EDAD A PARTIR DEL 1 DE ENERo de 1965

D A T O S B A S I C O S. 1-I-1961

\begin{tabular}{|c|c|c|c|c|c|c|c|c|c|c|}
\hline \multirow{2}{*}{ Años } & \multicolumn{2}{|c|}{ I-I-1961 } & \multicolumn{2}{|c|}{$1-I-1965$} & \multicolumn{2}{|c|}{$1-I-1970$} & \multicolumn{2}{|c|}{$31-X I I-1975$} & \multicolumn{2}{|c|}{$31 \cdot X I I-1980$} \\
\hline & Hombres & Mujeres & Hombres & Mujeres & Hombres & Mujeres & Hombres & Mujeres & Hombres & Mujeres \\
\hline $\begin{array}{cccccc}10 & \ldots & \ldots & \ldots & \ldots & \ldots \\
11 & \ldots & \ldots & \ldots & \ldots & \ldots \\
12 & \ldots & \ldots & \ldots & \ldots & \ldots \\
13 & \ldots & \ldots & \ldots & \ldots & \ldots \\
14 & \ldots & \ldots & \ldots & \ldots & \ldots \\
10-14 & \ldots & \ldots & \ldots & \ldots \\
& \ldots & \ldots & \ldots\end{array}$ & $\begin{array}{r}29 \\
28 \\
28 \\
28 \\
28 \\
141\end{array}$ & $\begin{array}{l}13 \\
12 \\
12 \\
11 \\
11 \\
59\end{array}$ & $\begin{array}{r}29 \\
29 \\
28 \\
28 \\
28 \\
142\end{array}$ & $\begin{array}{l}15 \\
14 \\
13 \\
12 \\
12 \\
66\end{array}$ & $\begin{array}{r}30 \\
29 \\
29 \\
28 \\
28 \\
144\end{array}$ & $\begin{array}{l}17 \\
16 \\
15 \\
14 \\
14 \\
76\end{array}$ & $\begin{array}{r}30 \\
29 \\
29 \\
29 \\
28 \\
146\end{array}$ & $\begin{array}{l}18 \\
18 \\
18 \\
17 \\
17 \\
88\end{array}$ & $\begin{array}{r}31 \\
30 \\
29 \\
29 \\
28 \\
148\end{array}$ & $\begin{array}{l}20 \\
20 \\
20 \\
20 \\
19 \\
99\end{array}$ \\
\hline $\begin{array}{cccccc}15 & \ldots & \ldots & \ldots & \ldots & \ldots \\
16 & \ldots & \ldots & \ldots & \ldots & \ldots \\
17 & \ldots & \ldots & \ldots & \ldots & \ldots \\
18 & \ldots & \ldots & \ldots & \ldots & \ldots \\
19 & \ldots & \ldots & \ldots & \ldots & \ldots \\
15-19 & \ldots & \ldots & \ldots & \ldots \\
15 & \ldots & \ldots & \ldots\end{array}$ & $\begin{array}{l}17 € \\
175 \\
175 \\
175 \\
174 \\
875\end{array}$ & $\begin{array}{r}68 \\
67 \\
67 \\
66 \\
65 \\
333\end{array}$ & $\begin{array}{l}178 \\
177 \\
176 \\
175 \\
175 \\
881\end{array}$ & $\begin{array}{r}\mathbf{7 6} \\
\mathbf{7 5} \\
\mathbf{7 5} \\
\mathbf{7 5} \\
\mathbf{7 5} \\
\mathbf{3 7 6}\end{array}$ & $\begin{array}{l}179 \\
179 \\
178 \\
178 \\
177 \\
890\end{array}$ & $\begin{array}{r}86 \\
86 \\
86 \\
86 \\
85 \\
429\end{array}$ & $\begin{array}{l}181 \\
181 \\
181 \\
180 \\
180 \\
903\end{array}$ & $\begin{array}{r}101 \\
100 \\
100 \\
100 \\
99 \\
500\end{array}$ & $\begin{array}{l}183 \\
183 \\
183 \\
182 \\
913\end{array}$ & $\begin{array}{l}113 \\
112 \\
112 \\
111 \\
560\end{array}$ \\
\hline $\begin{array}{llllll}20 & \ldots & \ldots & \ldots & \ldots & \ldots \\
21 & \ldots & \ldots & \ldots & \ldots & \ldots \\
22 & \ldots & \ldots & \ldots & \ldots & \ldots \\
23 & \ldots & \ldots & \ldots & \ldots & \ldots \\
24 & \ldots & \ldots & \ldots & \ldots & \ldots \\
20-24 & \ldots & \ldots & \ldots & \ldots \\
20 & \ldots & \ldots\end{array}$ & $\begin{array}{r}206 \\
206 \\
205 \\
205 \\
205 \\
1.027\end{array}$ & $\begin{array}{r}63 \\
63 \\
62 \\
62 \\
61 \\
311\end{array}$ & $\begin{array}{r}208 \\
208 \\
207 \\
206 \\
205 \\
1.034\end{array}$ & $\begin{array}{r}71 \\
70 \\
70 \\
70 \\
70 \\
\mathbf{3 5 1}\end{array}$ & $\begin{array}{r}211 \\
209 \\
209 \\
208 \\
207 \\
1.044\end{array}$ & $\begin{array}{r}81 \\
81 \\
80 \\
80 \\
79 \\
401\end{array}$ & $\begin{array}{r}214 \\
213 \\
212 \\
211 \\
210 \\
1.060\end{array}$ & $\begin{array}{r}94 \\
94 \\
93 \\
93 \\
93 \\
467\end{array}$ & $\begin{array}{r}216 \\
215 \\
214 \\
213 \\
1.071\end{array}$ & $\begin{array}{l}105 \\
105 \\
105 \\
104 \\
\\
\mathbf{5 2 3}\end{array}$ \\
\hline $\begin{array}{ccccc}25-29 & \ldots & \ldots & \ldots & \ldots\end{array}$ & 1.158 & 204 & 1.166 & 285 & 1.177 & 262 & 1.182 & 300 & 1.207 & 343 \\
\hline $\begin{array}{llllll}30-34 & \ldots & \ldots & \ldots & \ldots\end{array}$ & 1.136 & 201 & 1.144 & 171 & 1.135 & 260 & 1.183 & 307 & 1.185 & 337 \\
\hline $\begin{array}{ccccc}\mathbf{3 5}-39 & \ldots & \ldots & \ldots & \ldots \\
\mathbf{4 0 - 4 4} & \ldots & \ldots & \ldots & \ldots \\
\mathbf{4 5 - 4 9} & \ldots & \ldots & \ldots & \ldots \\
\mathbf{5 0 - 5 4} & \ldots & \ldots & \ldots & \ldots \\
\mathbf{5 5 - 5 9} & \ldots & \ldots & \ldots & \ldots \\
\mathbf{6 0 - 6 4} & \ldots & \ldots & \ldots & \ldots \\
\mathbf{6 5 - 6 9} & \ldots & \ldots & \ldots & \ldots \\
\mathbf{7 0 - 7 4} & \ldots & \ldots & \ldots & \ldots \\
\mathbf{7 5} & \text { y } & \ldots & \ldots & \ldots \\
\text { mas } & \ldots & \ldots & \ldots \\
\end{array}$ & $\begin{array}{l}954 \\
929 \\
793 \\
756 \\
615 \\
549 \\
221 \\
168 \\
190\end{array}$ & $\begin{array}{r}161 \\
159 \\
157 \\
152 \\
120 \\
112 \\
52 \\
43 \\
43\end{array}$ & $\begin{array}{l}960 \\
936 \\
800 \\
761 \\
620 \\
552 \\
222 \\
169 \\
192\end{array}$ & \begin{tabular}{r|}
183 \\
179 \\
177 \\
171 \\
135 \\
126 \\
59 \\
59 \\
48 \\
63
\end{tabular} & $\begin{array}{l}969 \\
946 \\
809 \\
768 \\
625 \\
558 \\
225 \\
170 \\
193\end{array}$ & $\begin{array}{r}208 \\
205 \\
202 \\
196 \\
163 \\
136 \\
67 \\
53 \\
72\end{array}$ & $\begin{array}{l}994 \\
949 \\
829 \\
771 \\
640 \\
560 \\
226 \\
175 \\
196\end{array}$ & $\begin{array}{r}243 \\
238 \\
235 \\
228 \\
177 \\
170 \\
78 \\
66 \\
82\end{array}$ & $\begin{array}{r}1.013 \\
951 \\
845 \\
772 \\
652 \\
561 \\
230 \\
175 \\
199\end{array}$ & $\begin{array}{r}272 \\
267 \\
263 \\
256 \\
196 \\
194 \\
87 \\
77 \\
90\end{array}$ \\
\hline TOTAL ... ... & 9.514 & 2.120 & 9.579 & 2.390 & 9.673 & 2.732 & 9.816 & 3.179 & 9.922 & 3.564 \\
\hline
\end{tabular}




\section{BIBLIOGRAFIA}

1. Avance de las «Clasificaciones de la Población», obtenido mediante una amuestra" del 1 por 100. Instituto Nacional de Estadística. Madrid, 1962.

2. «Poblaciones de derecho y hecho de los Municipios de la Nación». Censo de la Población y de las Viviendas, 1960. Instituto Nacional de Estadística. Madrid, 1962.

3. «Proyección de la Población de España en 1967 y 1970», Tomás Prieto Vilches. Revista del Instituto Nacional de Estadística: Estadística Española, núm. 20. Julio-septiembre 1963. Madrid.

4. «Movimiento nacional de la Población de España». Instituto Nacional de Estadística. Madrid, 1961.

5. «Esquemas de supervivencia de la Población española. Período 1957 a 1960». Instituto Nacional de Estadística. Madrid, 1964.

6. «Estudios migratorios, 1953-1964». Instituto Español de Emigración. Madrid, 1964.

7. «L'évolution démographique de 1956 à 1976 en Europe Occidentale et aux EtatsUnis». O. E. C. E., 1961.

8. «Estudio sobre la Población activa española». Dirección General de Empleo. Ministerio de Trabajo, 1964.

9. Censo de la Población de España. Año 1970. «Poblaciones de derecho y hecho de los Municipios». Instituto Nacional de Estadística. Madrid, 1971.

10. Informes de la O. C. D. E., núms. MO (64) 12 y MS/M/605/189.

11. «Proyección de la Población de España de 1965 a 1980», Dirección General de Empleo. Ministerio de Trabajo. Autor: Antonio Rodrfguez Socorro; colaboradores: Tomás Prieto V. y equipo técnico de la D. G. E., 1965.

12. Revista Información Comercial Española, núm. 496, diciembre 1974. Ministerio de Comercio. «Población». Artículo: «El futuro de la Población española. Perspectivas posibles", por Joaquín LeGUrNA.

13. "Tabla de mortalidad de funcionarios». M. P., 1962. Serie Estudios. Madrid, 1964. Instituto Nacional de Previsión.

14. «Población activa en 1972-73». Encuesta. Ministerio de Planificación del Desarrollo. I. N. E. Madrid, 1975. 
REVL-1976, núm. 189. RODRIGUEZ SOCORRO, ANTONIO. PROYECCION DE LA POBLACION NACI... $r$

\section{REVISTA}

DE

FSTUDIOS

DE LA $\mathrm{LA}$

S VIDA LOCAL

\section{JURISPRUDENCIA}


